\title{
Bénard instabilities in a binary-liquid layer evaporating into an inert gas
}

\author{
H. Machrafi, ${ }^{\mathrm{a},}$, A. Rednikov ${ }^{\mathrm{b}}$, P. Colinet ${ }^{\mathrm{b}}$, PC. Dauby ${ }^{\mathrm{a}}$
}

${ }^{a}$ Université de Liège, Thermodynamique des Phénomènes Irréversibles, Institut de Physique B5a, Allée du 6 Août, 17, B-4000 Liège 1, Belgium

${ }^{b}$ Université Libre de Bruxelles, TIPs - Fluid Physics, CP165/67, Avenue F.D. Roosevelt, 50, B-1050

Bruxelles, Belgium

\begin{abstract}
A linear stability analysis is performed for a horizontal layer of a binary liquid of which solely the solute evaporates into an inert gas, the latter being assumed to be insoluble in the liquid. In particular, a water-ethanol system in contact with air is considered, with the evaporation of water being neglected (which can be justified for a certain humidity of the air). External constraints on the system are introduced by imposing fixed "ambient" mass fraction and temperature values at a certain effective distance above the free liquid-gas interface. The temperature is the same as at the bottom of the liquid layer, where, besides, a fixed mass fraction of the solute is presumed to be maintained. Proceeding from a (quasi-)stationary reference solution, neutral (monotonic) stability curves are calculated in terms of solutal/thermal Marangoni/Rayleigh numbers as functions of the wavenumber for different values of the ratio of the gas and liquid layer thicknesses. The results are also presented in terms of the critical values of the liquid layer thickness as a function of the thickness of the gas layer. The solutal and thermal Rayleigh and Marangoni effects are compared to one another. For a water-ethanol mixture of $10 \mathrm{wt} \%$ ethanol, it appears that the solutal Marangoni effect is by far the most important instability mechanism. Furthermore, its global action can be described within a Pearson-like model, with an appropriately defined Biot number depending on the wavenumber. On the other hand, it is also shown that, if taken into account, water evaporation has only minor quantitative consequences upon the results for this predominant, solutal Marangoni mechanism.
\end{abstract}

\section{Keywords}

Stability analysis, binary mixture, evaporation, Rayleigh, Marangoni, solutal and thermal effects

\section{Introduction}

In standard studies [1-8] of convective instabilities of horizontal fluid layers, the destabilizing gradient across the layer is directly controlled from the outside of the system, through boundary conditions below and above the layer. The instability due to gravity and density variations is usually called Rayleigh-Bénard instability, while Marangoni-Bénard instability refers to the case where the surface tension variations are the driving factor behind it. When both effects couple, the term "Rayleigh-Marangoni-Bénard instability" is used. If it is the concentration dependence of the density and surface tension that causes the onset of convection, the corresponding instabilities can be called "solutal" Rayleigh-Marangoni-Bénard instabilities. Likewise, "thermal" instabilities result from the similar role of the temperature. 
When evaporation takes place at the upper surface of the liquid layer, the situation becomes even more intricate. Indeed, evaporation is an endothermic process, resulting into the cooling of the liquid surface. In the case of binary mixtures, it is also accompanied by concentration gradients across the layer. Since the density and surface tension depend on both the temperature and the concentration, evaporation can thus indirectly destabilize the liquid layer.

Convection due to evaporation is an important phenomenon that occurs in many applications, such as during the drying of paint films, coatings, heat exchangers and process engineering installations. It also occurs in nature when for instance a salty lake dries out due to the evaporation of water, leaving behind structures on the soil. Several theoretical works have already been published on evaporation-driven Bénard instability of a one-component liquid layer [9-15], with the liquid evaporating in either an inert gas [9-13] or in its own vapor [6, 14-15]. To our knowledge, the studies of two-component systems has been rather limited in this context. One can mention a scaling analysis [16] or experiments [17]. From the theoretical viewpoint, quite a comprehensive study has been carried out in the case of a spherical geometry, when the Marangoni (both thermal and solutal) instability has been considered for an evaporating binaryliquid droplet [18]. Let us also mention experiments in a Hele-Shaw cell configuration with evaporating water-alochol solutions [19,20], where density-fingering (plume-like) patterns have been observed after a certain time had elapsed since the exposure of the solution to the air, which is clearly a manifestation of a buoyancy-driven mechanism. Cellular Marangoni patterns have also been observed [19], which are then suppressed by adding a surfactant, and the theoretical part of [19] concerns just the buoyancy-driven instability.

In the present paper, the Rayleigh-Marangoni-Bénard instability induced by evaporation is studied by means of a linear stability analysis in the case of a binary liquid layer, when both the solutal and the thermal factors are involved. The particular model used here assumes a dilute solution of which only the solute evaporates (even though the latter limitation is relaxed at a later stage). In this case, the gas layer consists of an inert gas and the vapor of the evaporating liquid. The aim of the paper is to study the different instability mechanisms and to assess the degree of their mutual importance using a configuration as simple as possible. A concrete example followed throughout the paper is a $10 / 90 \mathrm{wt} \%$ ethanol/water mixture at normal conditions.

The paper is organized as follows. In section 2, the studied configuration is described, and a mathematical formulation of the problem is provided assuming that it is only the solute (ethanol) that evaporates from the binary mixture. The reference state is considered in section 3 . Section 4 is concerned with the formulation of the linear stability problem. The results of the linear stability analysis are presented in section 5, and the relative importance of various instability mechanisms is assessed. An approximate analytical treatment of the reference profile and of the marginal stability conditions (by means of a Pearson-like model) is carried out in section 6, 
making use of various simplifications possible within the full model. In section 7 , the model is generalized to account for solvent (water) volatility, although the analysis is limited to the approximate approach framework of section 6. Finally, the conclusions are summarized in section 8 .

\section{Description of the problem}

The system the instability of which is studied in this paper is presented in Fig. 1. It consists of a binary liquid layer (thickness $d_{l}$, also denoted $h^{d}$, see the distinction between the two below) in contact with a gas layer (thickness $d_{g}$, also denoted $\left(H^{d}-h^{d}\right)$, so that $H^{d}$ is the total thickness of the two-layer system). The liquid layer rests on a horizontal solid surface with a fixed temperature. The liquid-gas interface is assumed to be undeformable. The liquid layer is made up of a solute in dilute concentration and a solvent. The gas layer consists of air (the absorption of which in the liquid is neglected) and the vapors of the solute and the solvent.

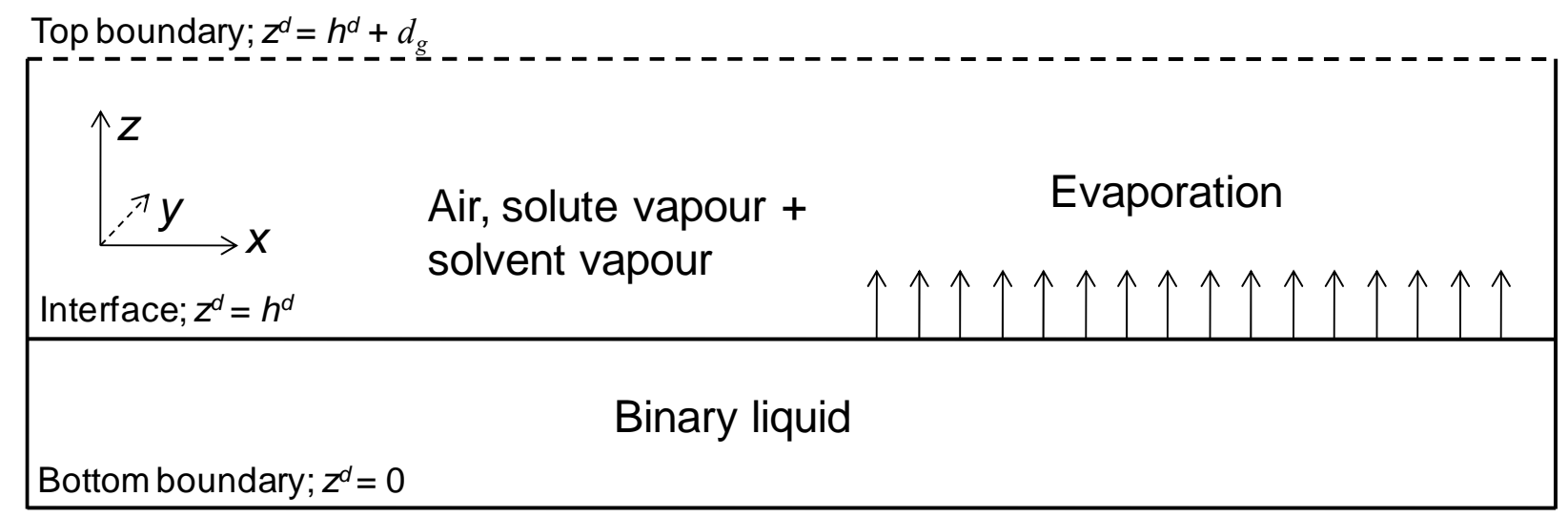

Fig. 1. Sketch of the studied system

The treatment of the gas layer adopted here follows Haut and Colinet [10]. The thickness $d_{g}$ in such an approach is just viewed as a semi-heuristic quantity describing the typical equivalent (effective) diffusion length in the gas phase as determined by external air currents which may be naturally present or deliberately created (ventilation) therein: $d_{g}$ is the distance at which the diffusion is formally of the same magnitude as the convective transport in a real setup. In this sense, the gas phase above this layer is considered as perfectly mixed while ensuring given "ambient" values of temperature (the same as at the bottom of the liquid layer in the case considered here) and concentration at the effective upper boundary of our gas layer. In this respect, the approach is actually rather similar to the so-called "stagnant film" approach, often used in chemical engineering [21]. The shear-induced influence of such currents on the liquid layer is neglected however, and thus no net horizontal flow in the gas phase is explicitly included into the model. Besides, the model is formulated assuming no externally imposed horizontal nonuniformities upon the system, implying that the scale of any horizontal non-uniformity that may exist in a real setup (as opposed to the present idealized configuration) is much greater than the 
scale of the phenomena to be studied here (evaporation-induced Bénard instability). As for the hydrodynamic conditions at the effective upper boundary of the gas layer, we shall use the "soft" ("stress-free") ones: no tangential stress and a given uniform pressure/normal stress. We note that, overall, despite its heuristic character, the proposed approach is more detailed and general than the frequently used one based upon describing the transport processes in the gas by means of simply a transfer coefficient (Biot number): the former permits to assess an active role of the gas phase in the studied phenomena, whereas the latter (being essentially a one-sided model of the liquid layer) does not.

The solvent is considered to be much less volatile than the solute. Actually, in the main body of the paper (sections 2-6), the solvent is formally treated as non-volatile. For a dilute solution of ethanol (solute) in water (solvent), which is a concrete example followed throughout the paper, such a treatment is expected to be approximately valid at some ambient humidity of the air, when the water vapor is nearly saturated relative to the solution (otherwise, even though water is indeed much less volatile than ethanol, its greater amount in the solution may make the effects of its evaporation nonetheless noticeable). At the end of the paper, however, we shall come back to the question of how the results change if water evaporation is incorporated into the model for an arbitrary humidity of the air.

Here we shall also assume that it is not only the temperature that is fixed at the bottom of the liquid layer, but also the concentration. While the latter assumption seems to be rather artificial, it will permit to study in a simple way (i.e. for a quasi-stationary reference state) the Bénard instability mechanisms pertinent to an evaporating binary-liquid layer and to assess their mutual importance, which is the main goal of the present paper.

Now a few words about the notations $d_{l}, d_{g}, h^{d}$ and $H^{d}$ as used here. Due to evaporation, the liquid thickness changes with time $t^{d}$, which we describe here by introducing the function $h^{d}=h^{d}\left(t^{d}\right)$. We can then define a constant quantity $d_{l}$ as the thickness of the liquid layer at a certain reference, or initial, time $t^{d}=t_{0}^{d}$, i.e. $d_{l} \equiv h^{d}\left(t_{0}^{d}\right)$. This thickness $d_{l}$ will then be chosen as the unit length for non-dimensionalization. In dimensionless form, the bottom plate is located at $z=0$, and the interface then corresponds to $z=h(t)$, with $h\left(t_{0}\right)=1$. Note that the superscript " $d$ " stands for the dimensional character a particular quantity, whenever it is used elsewhere in dimensionless form. Similarly, in view of the meaning attributed to the gas layer thickness here, $H^{d}$ (defined as the total thickness of the system) can in principle also be a function of time: $H^{d}=H^{d}\left(t^{d}\right)$. Then we define $d_{g} \equiv H^{d}\left(t_{0}^{d}\right)-h^{d}\left(t_{0}^{d}\right)$ for the initial thickness of the gas layer. In dimensionless form, the top of the gas phase corresponds to $z=H(t)$.

\subsection{Bulk equations}

The Boussinesq approximation [6] will be adopted for both phases of the system, implying that the material properties of the fluids are treated as constant except for the density in the buoyancy 
terms, whose dependence on the temperature and mass fraction is taken in the following linearized form:

$\rho_{l}=\rho_{l, 0}\left\{1-\alpha_{l}\left(T_{l}^{d}-T_{l, 0}^{d}\right)-\varepsilon_{l}\left(c_{l}-c_{l, 0}\right)\right\}$

$\rho_{g}=\rho_{g, 0}\left\{1-\alpha_{g}\left(T_{g}^{d}-T_{g, 0}^{d}\right)-\varepsilon_{g}\left(c_{g}-c_{g, 0}\right)\right\}$,

whereas the corresponding dependence on pressure is presumed to be negligible for the pressure range involved in the problem. Here, $\rho$ is the density, $T^{d}$ is the (dimensional) temperature, $c$ is the solute mass fraction, $\alpha$ and $\varepsilon$ are the thermal and the solutal expansion coefficients. The subscripts " $l$ " and " $g$ " relate to the liquid and gas phases, respectively. The subscript " 0 " refers to a certain reference state (to be specified later on). Clearly, the validity of different hypotheses underlying the Boussinesq approximation is limited to situations for which the temperature and mass fraction in the system remain close enough to the reference values introduced in (1) and (2).

As mentioned above, let $d_{l}$ be the length scale. The time scale is chosen as the liquid thermal time scale $d_{l}^{2} / \kappa_{l}$. The dimensionless evaporation mass flux $J$ is obtained by using the scale $\frac{\lambda_{l} \theta}{L d_{l}}=\frac{\kappa_{l} \rho_{l}}{E d_{l}}$, where

$E=\frac{L}{\theta c_{p, l}}$

is the evaporation number, $\theta$ is the temperature scale, $L$ is the heat of solution of the solute in the solvent, $\kappa$ refers to the thermal diffusivity, $c_{p}$ the heat capacity and $\lambda$ the thermal conductivity. We shall choose the temperature scale such that

$\theta=\frac{L}{c_{p, l}} \quad(E \equiv 1)$.

However, we find it advantageous, for the sake of physical clarity of certain formulae, to keep the quantity $\theta$ unsubstituted, even though it will eventually be evaluated according to the above expression. The dimensionless temperatures $T_{l}$ and $T_{g}$ in the liquid and gas are respectively defined by $\left(T_{l}^{d}-T_{l, 0}^{d}\right) / \theta$ and $\left(T_{g}^{d}-T_{g, 0}^{d}\right) / \theta$. The mass fractions are already dimensionless. The pressure and velocity scales are respectively chosen as $\frac{\mu_{l} \kappa_{l}}{d_{l}^{2}}$ and $\frac{\kappa_{l}}{d_{l}}$, where $\mu$ refers to the dynamic viscosity. The following dimensionless balance equations are then obtained:

$\nabla \cdot \vec{v}_{l}=0$

$\frac{\partial \vec{v}_{l}}{\partial t}=-\left(\vec{v}_{l} \cdot \vec{\nabla}\right) \vec{v}_{l}+P r_{l}\left\{-\vec{\nabla} p_{l}-G a_{l} 1_{z}+\nabla^{2} \vec{v}_{l}+R a_{l} T_{l} 1_{z}+R s_{l} L e_{l}\left(c_{l}-c_{l, 0}\right) 1_{z}\right\}$,

$\frac{\partial T_{l}}{\partial t}=-\left(\vec{v}_{l} \cdot \vec{\nabla}\right) T_{l}+\nabla^{2} T_{l}$,

$\frac{\partial c_{l}}{\partial t}=-\left(\vec{v}_{l} \cdot \vec{\nabla}\right) c_{l}+L e_{l}\left\{\nabla^{2} c_{l}+\psi_{s} \nabla^{2} T_{l}\right\}$

$\nabla \cdot \vec{v}_{g}=0$

$\frac{\partial \vec{v}_{g}}{\partial t}=-\left(\vec{v}_{g} \cdot \vec{\nabla}\right) \vec{v}_{g}+P r_{l}\left\{-\rho^{-1} \vec{\nabla} p_{g}-G a_{l} 1_{z}+v \nabla^{2} \vec{v}_{g}+\alpha R a_{l} T_{\mathrm{g}} 1_{z}+\varepsilon R s_{l} L e_{l}\left(c_{g}-c_{g, 0}\right) 1_{z}\right\}$,

$\frac{\partial T_{g}}{\partial t}=-\left(\vec{v}_{g} \cdot \vec{\nabla}\right) T_{g}+\kappa \nabla^{2} T_{g}$,

$\frac{\partial c_{g}}{\partial t}=-\left(\vec{v}_{g} \cdot \vec{\nabla}\right) c_{g}+D L e_{l} \nabla^{2} c_{g}$, 
where $\vec{v}$ and $p$ are the (barycentric) velocity and pressure fields. For the liquid and gas phase respectively, (3) and (7) are the continuity equations for incompressible fluids, (4) and (8) are the momentum equations, (5) and (9) express the energy conservation, whereas (6) and (10) stand for the mass conservation of the solute species. The symbol $\psi_{S}$ accounts for the Soret effect (considered only in the liquid phase) and is defined by

$\psi_{s}=S_{l, 0} \theta$ with $S_{l, 0}=\frac{D_{T, l}}{D_{l}} c_{l, 0}\left(1-c_{l, 0}\right)$,

where $D_{T}$ is the thermal diffusion coefficient and $D$ refers to the diffusion coefficient. $S_{l, 0}$ is the Soret coefficient in the liquid (obtaining a value $\psi_{s} \approx 0.154$ ). In the gas phase, the Soret effect is neglected assuming small vapor concentration, in which limit this effect tends to disappear (and the same goes for the Dufour effect). Equations (3) through (10) contain the following dimensionless numbers:

$P r_{l}=\frac{v_{1}}{\kappa_{l}}, L e_{l}=\frac{D_{l}}{\kappa_{l}}, G a_{l}=\frac{g d_{l}^{3}}{\kappa_{l} v_{l}}, R a_{l}=\frac{\alpha_{l} g \theta d_{l}^{3}}{\kappa_{l} v_{l}}, R s_{l}=\frac{\varepsilon_{l} g d_{l}^{3}}{D_{l} \nu_{l}}, \rho=\frac{\rho_{g}}{\rho_{l}}, v=\frac{v_{g}}{v_{l}}, \alpha=\frac{\alpha_{g}}{\alpha_{l}}, \varepsilon=\frac{\varepsilon_{g}}{\varepsilon_{l}}$, $\kappa=\frac{\kappa_{g}}{\kappa_{l}}, D=\frac{D_{g}}{D_{l}}$,

where $v$ refers to the kinematic viscosity and $P r_{l}, L e_{l}, G a_{l}, R a_{l}$ and $R s_{l}$ are respectively the Prandtl, Lewis, Galileo, thermal Rayleigh and solutal Rayleigh numbers in the liquid. The symbols $\rho, v, \alpha, \varepsilon, \kappa$ and $D$, without subscripts, denote the ratios of the corresponding material properties in the gas to those in the liquid.

For the subsequent discussions in the paper, it is also interesting to recall the following detailed dimensionless expressions of the solute mass and heat fluxes in the liquid and in the gas compatible with (5), (6), (9) and (10):

$\vec{J}_{l}=-L e_{l}\left\{\nabla c_{l}+\psi_{s} \nabla T_{l}\right\}, \quad \vec{q}_{l}=-\nabla T_{l}, \quad \vec{J}_{g}=-\rho D L e_{l} \nabla c_{g} \quad \vec{q}_{g}=-\lambda \nabla T_{g}$.

Note that the scales used for the mass fluxes are the same as the one defined above for the evaporation flux (with $E \equiv 1$ ). For the heat flux, the scale used is $\frac{\lambda_{l} \theta}{d_{l}}$.

\subsection{Boundary conditions}

The following boundary conditions are assumed at the bottom of the liquid layer $(z=0)$ : $\vec{v}_{l}=0, \quad T_{l}=T_{b}, \quad c_{l}=c_{b}$,

where $T_{b}$ and $c_{b}$ are some fixed values. Note that the boundary condition $c_{l}=c_{b}$ on the mass fraction is probably not easy to realize in practice. In principle, this condition could be realized by installing at the bottom a thin layer of a porous gel (of vanishing mass transfer resistance), under which a mixture of the solvent and the solute, with a fixed mass fraction $c_{b}$, is circulated. In the present paper, as it has already been mentioned, we shall rather regard the third condition (13) as a model permitting to study in a simple way the basic instability mechanisms in a binaryliquid layer. In particular, it will allow consideration of a (quasi-)steady reference state (which would not be the case should a zero flux condition $\vec{J}_{l}=0$ be specified instead). 
The boundary conditions to be specified at the top of the gas layer have in fact been already discussed when introducing the basic configuration. We have fixed constant values of the temperature and concentration, $T_{t}$ and $c_{t}$, respectively, and the "soft" hydrodynamic conditions with the normal stress being equal to a certain value $p_{t}$ (an "external pressure") and the tangential stress being zero ("stress free condition"). Thus,

$T_{g}=T_{t}, \quad c_{g}=c_{t}, \quad \frac{\partial u_{g}}{\partial z}+\frac{\partial w_{g}}{\partial x}=\frac{\partial v_{g}}{\partial z}+\frac{\partial w_{g}}{\partial y}=0, \quad-p_{g}+2 \mu \frac{\partial w_{g}}{\partial z}=-p_{t}$,

at $z=H$, where

$\mu=\frac{\mu_{g}}{\mu_{l}}$

is the ratio of the dynamic viscosities. The symbols $u, v$ and $w$ stand for the $x, y$ and $z$ velocity field components, respectively. It will be seen that the form of the hydrodynamic conditions has little influence in practice (see subsection 5.7).

Consider now the conditions at the liquid-gas interface. Since evaporation takes place at this interface, the liquid layer thickness changes with time, but we assume that the interface remains flat. Note that this assumption will be discussed briefly at the end of section 6.2. Due to mass conservation, the evaporation flux $J$ is linked to velocities in both the gas phase and the liquid phase in the reference frame moving with the interface $z=h(t)$. In our notations, this reads [6]

$J=\left(w_{l}-\frac{d h}{d t}\right)=\rho\left(w_{g}-\frac{d h}{d t}\right)$,

at $z=h(t)$. Note that (15) can also be solved with respect to $J$ and $d h / d t$ to yield

$\frac{d h}{d t}=\frac{1}{1-\rho}\left(w_{l}-\rho w_{g}\right)$,

$J=\frac{\rho}{1-\rho}\left(w_{g}-w_{l}\right)$

at $z=h(t)$.

Other boundary conditions at the interface express the continuity of the temperature and of the tangential velocity components :

$T_{g}=T_{l}, \quad u_{l}=u_{g}, \quad v_{l}=v_{g}$,

at $z=h(t)$.

The energy conservation at the interface implies a balance between the heat fluxes in both phases and the heat of evaporation, which reads [6]

$-\frac{\partial T_{l}}{\partial z}+\lambda \frac{\partial T_{g}}{\partial z}=J$

at $z=h(t)$, where

$\lambda=\frac{\lambda_{g}}{\lambda_{l}}$.

Note that the mechanical effects (kinetic energy flux and work of surface tension forces) are neglected in (19). 
In expressing the tangential stress balance at the interface, it is assumed that the surface tension between the liquid and the gas depends on the temperature and on the solute mass fraction in the liquid. Similarly to (1) and (2), one can write

$\gamma=\gamma_{0}-\gamma_{T}\left(T_{l}^{d}-T_{l, 0}^{d}\right)-\gamma_{C}\left(c_{l}-c_{l, 0}\right)$

with

$\gamma_{T}=-\left(\frac{\partial \gamma}{\partial T}\right)$ and $\gamma_{C}=-\left(\frac{\partial \gamma}{\partial c}\right)$.

Then, the conditions expressing the tangential stress balance at $z=h(t)$ are [6]

$-\mu\left(\frac{\partial w_{g}}{\partial x}+\frac{\partial u_{g}}{\partial z}\right)+\left(\frac{\partial w_{l}}{\partial x}+\frac{\partial u_{l}}{\partial z}\right)+M a \frac{\partial T_{l}}{\partial x}+M s L e \frac{\partial c_{l}}{\partial x}=0$,

$-\mu\left(\frac{\partial w_{g}}{\partial y}+\frac{\partial v_{g}}{\partial z}\right)+\left(\frac{\partial w_{l}}{\partial y}+\frac{\partial v_{l}}{\partial z}\right)+M a \frac{\partial T_{l}}{\partial y}+M s L e \frac{\partial c_{l}}{\partial y}=0$

where

$M a=\frac{\gamma_{T} d_{l} \theta}{\kappa_{l} \mu_{l}}, M s=\frac{\gamma_{C} d_{l}}{\mathrm{D}_{1} \mu_{l}}$

are the thermal and solutal Marangoni numbers.

The assumption that it is only the solute that evaporates implies that the evaporation flux $J$ is equal to the evaporation flux of the solute only. The latter can be expressed as:

$J c_{l}+\overrightarrow{J_{l}} \cdot 1_{z}$.

By equating this to $J$ and using (12), one arrives at the following boundary condition at $z=h(t)$ :

$J=-\frac{L e_{l}}{1-c_{l}}\left\{\frac{\partial c_{l}}{\partial z}+\psi_{S} \frac{\partial T_{l}}{\partial z}\right\}$.

The inert gas adsorption in the liquid is also neglected. Using an argument similar to the one leading to (23), one obtains the following additional boundary condition at $z=h(t)$ :

$J=-\frac{\rho D L e_{l}}{1-c_{g}} \frac{\partial c_{g}}{\partial z}$.

A local equilibrium hypothesis at the surface is made to describe the evaporation process. Assuming a dilute binary liquid, it amounts to the so-called Henry's law [22]:

$y_{g} p_{g}^{d}=y_{l} K_{e}$,

where $y_{g}$ and $y_{l}$ are the molar fractions of the solute in the gas and liquid phases, respectively, and $K_{e}$ is the Henry coefficient (in pressure units), which generally depends on the interface temperature, even though this dependence is neglected in the present paper (this point will be justified a posteriori in Section 3, in the case of the reference solution). The quantity $p_{g}^{d}$ is the total pressure of the gas at the interface. In terms of mass fractions, Henry's law can be rewritten as

$\frac{c_{g} \delta_{M}^{\prime \prime}}{1+c_{g}\left(\delta_{M}^{\prime \prime}-1\right)}=\frac{c_{l} \delta_{M}}{1+c_{l}\left(\delta_{M}-1\right)} \frac{K_{e}^{n}}{p_{g}}$

yielding another boundary condition at $z=h(t)$. The symbol $K_{e}^{n}$ stands for the dimensionless Henry coefficient (using $\mu_{l} \kappa_{l} / d_{l}^{2}$ as the pressure scale). The symbol $\delta$ refers to the ratio of solvent and solute properties, the subscript of $\delta$ indicating the property in question. In (25) 
$\delta_{M}=\frac{M_{1}}{M_{2}}$

is the solvent to solute molecular mass ratio, while

$\delta_{M}^{\prime \prime}=\frac{M_{a}}{M_{2}}$

is the same for the air and the solute, where the subscript " $a$ " refers to the air. Note also the notation

$\delta_{M}^{\prime}=\frac{M_{a}}{M_{1}}=\delta_{M}^{\prime \prime} / \delta_{M}$

which will be used later on.

\subsection{Comments on the linear stability analysis}

The goal here is to examine the stability of the horizontally uniform solutions of the above equations, depending only on the vertical coordinate and characterized by zero horizontal velocity (the "reference" solutions). It should be realized, however, that due to the evaporation process at the liquid-gas interface, the thickness of the liquid layer will vary in the course of time. Consequently, the possible horizontally uniform solutions will be intrinsically timedependent. However, the analysis is carried out assuming this variation to be slow enough (the quasi-stationary hypothesis, to be defined shortly). As a first step of our work, we obtain the (quasi-stationary) reference solution. The second step will consist in analyzing the stability of the reference solution. Since the latter depends on time, it must of course be understood that the stability analysis must be carried out as a function of time. This is accomplished in the framework of the frozen-time approach (also used in [23]), which is in any way nearly rigorous here on account of the mentioned quasi-stationary hypothesis (with the exception of certain modes as discussed later on).

More precisely, the quasi-stationary assumption consists in assuming that the liquid thickness $h(t)$ varies sufficiently slowly for the temperature, mass fraction and velocity profiles in both phases to reach a steady state corresponding to an instantaneous value of $h(t)$. In other words, this hypothesis amounts to the assumption that the time scale of the variations of $h^{d}=h^{d}\left(t^{d}\right)$ is much larger than the diffusive time scales of the problem. To determine the quasi-stationary reference solution, all time derivatives in the equations can be cancelled, except for the time derivative of $h$ in (16). It goes without saying that this ansatz is not valid for very short times, when the diffusional profiles have not yet invaded the whole layer. As a consequence of this quasi-stationary assumption and the corresponding separation of timescales, it is easy to understand that among all the unknowns of the problem, only $h$ will explicitly depend on time, following (16), while all other quantities of the reference solution will depend on $t$ only through $h$ itself. Note that this provides the reason why the approach is called quasi-stationary. As a further interesting consequence of this hypothesis, one can understand that performing a frozentime stability analysis of the reference solution for all times $t$ is completely equivalent to performing the stability analysis for all possible values of $h$. Switching to this point of view amounts to asking for what $h$ an instability can occur, instead of asking for what time this 
instability appears, which is often more interesting from a physical point of view and which will actually be done in the following.

In the framework of this approach, the time $t_{0}^{d}$ introduced above can be chosen, without loss of generality, as the time corresponding to the thickness $h^{d}\left(t_{0}^{d}\right)$ for which the stability analysis shall be performed. Following the procedure described before, to non-dimensionalize the equations, one deduces that the dimensionless liquid thickness can be set to unity

$h=1$.

Note that this result does not imply that the dimensionless thickness is fixed, but only means that its value, at the time for which a stability analysis will be carried out, is equal to unity. Let us also stress that replacing the dimensionless liquid thickness by unity is a bit delicate, since this amounts to hide that the reference solution indirectly depends on time through $h$ and to transfer this time dependence in the length scale used to non-dimensionalize the equations. Fortunately, this will not prevent a proper stability analysis, as we will explain later.

In principle, in accordance with the approach used for the gas layer in our system, as set forth in the beginning of section 2, $H$ can also be a function of time. Furthermore, unlike $h$ whose time dependence is embedded into the formulation by means of (16), for $H$, it is largely controlled by the external constraints on the system which remain outside the present formulation. For instance, for constant external constraints, it may be reasonable to assume that it is $(H-h) d_{l}$ (the dimensional gas layer thickness) that remains constant, but then $H$ will evolve together with $h$. In any event, the time dependence of $H$ (if any) will be presumed as slow as that of $h$.

\subsection{Water-ethanol system and external constraints}

Before undertaking the stability analysis, it is important to stress that a very large number of parameters appear in the equations presented above and that it is not feasible to carry out a general parametric study. For this reason, we shall first restrict our analysis to a water-ethanol liquid layer, with only ethanol evaporating into air. Then, we shall assume that the temperatures imposed at the bottom and at the top of the system are equal. In this way, any thermal instabilities that may appear in the system would result exclusively from the evaporation process. The reference temperatures in both the liquid and the gas phases that are used in (1), (2) and (20) are then fixed at this value, i.e. $T_{l, 0}^{d}=T_{g, 0}^{d}=T_{t}^{d}=T_{b}^{d}$. This means that we have just $T_{b}=T_{t}=0$ in dimensionless form.

We shall also choose the mass fraction $c_{b}$ imposed at the bottom of the system as the typical concentration $c_{l, 0}$, introduced in (1), (11) and (20). Similarly, the concentration $c_{t}$ imposed at the top of the system is used as the typical concentration $c_{g, 0}$ introduced in (2). The value $c_{b}$ must be small enough for Henry's law, corresponding to dilute solutions, to be actually valid. In particular, the following values are chosen: $T_{b}^{d}=T_{t}^{d}=300 \mathrm{~K}, c_{t}=0$. For the mass fraction imposed at the bottom, two different values are considered in our results, namely $c_{b}=0.1$ and 
$c_{b}=0.001$. The different thermo-physical properties of the liquid used in the calculations are those corresponding to the reference values of the temperature and composition imposed at the bottom of the system. Similarly, in the gas, the thermo-physical properties correspond to the reference temperature and composition imposed at the top of the upper layer. When needed, the properties are also determined for a value of the pressure equal to the atmospheric pressure $p_{t}^{d}=1 \mathrm{~atm}$.

The numerical values of the physical properties are given in tables 1 and 2, and for the interested reader, Appendix A presents some details on how these values have been obtained.

Table 1. Physical properties of the gas layer

\begin{tabular}{cl}
\hline \hline Physical property & Value \\
\hline$\rho_{g}$ & $1.18 \mathrm{~kg} / \mathrm{m}^{3}$ \\
$c_{p, g}$ & $1.005^{*} 10^{3} \mathrm{~J} / \mathrm{kg} \mathrm{K}$ \\
$\lambda_{g}$ & $2.62 * 10^{-2} \mathrm{~W} / \mathrm{m} \mathrm{K}$ \\
$\kappa_{g}$ & $2.22 * 10^{-5} \mathrm{~m}^{2} / \mathrm{s}$ \\
$v_{g}$ & $1.58 * 10^{-5} \mathrm{~m}^{2} / \mathrm{s}$ \\
$\mu_{g}$ & $1.85 * 10^{-5} \mathrm{~Pa}^{*} \mathrm{~s}$ \\
$\alpha_{g}$ & $3.35 * 10^{-3} \mathrm{~K}^{-1}$ \\
$D_{g}$ & $1.20 * 10^{-5} \mathrm{~m}^{2} / \mathrm{s}$ \\
$\varepsilon_{g}$ & $-3.70^{*} 10^{-1}$ \\
\hline \hline
\end{tabular}

Table 2. Physical properties of the liquid layer

\begin{tabular}{ccc}
\hline \hline Physical property & Value for $c_{b}=0.1$ & Value for $c_{b}=0.001$ \\
\hline$\rho_{l}$ & $9.796^{*} 10^{2} \mathrm{~kg} / \mathrm{m}^{3}$ & $9.964^{*} 10^{2} \mathrm{~kg} / \mathrm{m}^{3}$ \\
$c_{p, l}$ & $4.26^{*} 10^{3} \mathrm{~J} / \mathrm{kg} \mathrm{K}$ & $4.18^{*} 10^{3} \mathrm{~J} / \mathrm{kg} \mathrm{K}$ \\
$\lambda_{l}$ & $5.31^{*} 10^{-1} \mathrm{~W} / \mathrm{m} \mathrm{K}$ & $6.07 * 10^{-1} \mathrm{~W} / \mathrm{m} \mathrm{K}$ \\
$\kappa_{l}$ & $1.27 * 10^{-7} \mathrm{~m}^{2} / \mathrm{s}$ & $1.46^{*} 10^{-7} \mathrm{~m}^{2} / \mathrm{s}$ \\
$v_{l}$ & $1.33^{*} 10^{-6} \mathrm{~m}^{2} / \mathrm{s}$ & $8.5^{*} 10^{-7} \mathrm{~m}^{2} / \mathrm{s}$ \\
$\mu_{l}$ & $1.3 * 10^{-3} \mathrm{~Pa}^{*} \mathrm{~s}$ & $8.5^{*} 10^{-4} \mathrm{~Pa}^{*} \mathrm{~s}$ \\
$\alpha_{l}$ & $3.4^{*} 10^{-4} \mathrm{~K}^{-1}$ & $2.75^{*} 10^{-4} \mathrm{~K}^{-1}$ \\
$D_{l}$ & $1.0^{*} 10^{-9} \mathrm{~m}^{2} / \mathrm{s}$ & $1.3 * 10^{-9} \mathrm{~m}^{2} / \mathrm{s}$ \\
$\varepsilon_{l}$ & $1.53^{*} 10^{-1}$ & $1.92^{*} 10^{-1}$ \\
$\gamma_{T}$ & $1.45^{*} 10^{-4} \mathrm{~N} / \mathrm{m} \mathrm{K}$ & $1.6^{*} 10^{-4} \mathrm{~N} / \mathrm{m} \mathrm{K}$ \\
$\gamma_{C}$ & $1.4 * 10^{-1} \mathrm{~N} / \mathrm{m}$ & $5.0^{*} 10^{-1} \mathrm{~N} / \mathrm{m}$ \\
$K_{e}$ & $3.26^{*} 10^{-1} \mathrm{~atm}$ & $3.26^{*} 10^{-1} \mathrm{~atm}$ \\
$L$ & $1.13^{*} 10^{6} \mathrm{~J} / \mathrm{kg}$ & $1.13^{*} 10^{6} \mathrm{~J} / \mathrm{kg}$ \\
$D_{T, l} / D_{l}$ & $6.46^{*} 10^{-3} \mathrm{~K}$ & $7.58^{-1} 10^{-3} \mathrm{~K}$ \\
\hline \hline
\end{tabular}




\section{The reference state}

In the present section, we will determine the reference solution, the stability of which will be examined later on. We shall do it in the framework of the quasi-stationary assumption as discussed in subsection 2.3.

In seeking the reference solution, the partial time derivatives are neglected in the balance equations (3)-(10). When the horizontal derivatives are also disregarded, one obtains a system of ordinary differential equations with respect to the vertical coordinate $z$. Using the boundary conditions at $z=0,1$ and $H$, these equations can be solved, as now explained.

The first of the boundary conditions (13) together with the incompressibility assumption (3) directly yields the reference solution for the velocity in the liquid phase:

$w_{l, \text { ref }}=0$.

Similarly, equations (7) and (17) yield

$w_{g, r e f}=\frac{(1-\rho)}{\rho} J_{\text {ref }}$,

whereas note that

$\dot{h}=-J_{\text {ref }}$

according to (16). Using (5), the second of Eqs (13) and Eq. (26) yields the following reference solution for the temperature in the liquid phase:

$T_{l, r e f}=\left(T_{i, r e f}-T_{b}\right) z+T_{b}$,

where the subscript " $i$ " refers to the interface, and where the reference interfacial temperature $T_{i, r e f}$ will be determined below. Similarly, equation (9) and the first condition (14) yield

$T_{g, r e f}=T_{i, r e f}+\left(T_{t}-T_{i, r e f}\right) \frac{\left.1-e^{\left\{w_{g, r e f}(\mathrm{z}-1) / \kappa\right.}\right\}}{1-e^{\left\{w_{g, r e f}(H-1) / \kappa\right\}}}$.

Proceeding similarly for the mass fractions, by using (6), (10), the third of Eqs (13) and the second of Eqs (14), one obtains

$c_{l, r e f}=\left(c_{i, r e f, l}-c_{b}\right) z+c_{b}$,

$c_{g, r e f}=c_{i, r e f, g}+\left(c_{t}-c_{i, r e f, g}\right) \frac{\left.1-e^{\left\{w_{g, r e f}(z-1) /\left(D L e_{l}\right)\right.}\right\}}{\left.1-e^{\left\{w_{g, r e f}(H-1) /\left(D L e_{l}\right)\right.}\right\}}$.

In what follows, we will also need the pressure field in the gas in order to use it in Henry's law. From (8) together with (30), (32) and the last equation (14), one obtains the gas pressure distribution in the reference state, $p_{g, r e f}$. In particular, its value at the interface $(z=1)$ is found to be

$p_{i, r e f, g}=$

$p_{t}+\rho(1-H)\left[-G a_{l}+\alpha R a_{l}\left(T_{i, r e f}+\frac{\left(T_{t}-T_{i, r e f}\right)}{\left.1-e^{\left\{w_{g}, r e f\right.}(H-1) / \kappa\right\}}\right)+\varepsilon R s_{l} L e_{l} \frac{\left(c_{i, r e f, g} c_{t}\right)}{\left.1-e^{-\left\{w_{g, r e f}(H-1) / D L e_{l}\right\}}\right]}\right]-\rho\left[\frac{\alpha R a_{l} \kappa}{w_{g, r e f}}\left(T_{t}-T_{i, r e f}\right)+\right.$ $\left.\frac{\varepsilon R s_{l} D L e_{l}^{2}}{w_{g, r e f}}\left(c_{t}-c_{i, r e f, g}\right)\right]$.

In (29), (30) and (33), $T_{b}$ and $T_{t}$ denote respectively the temperature at the bottom and top plates. These are equal to one another as stipulated earlier (cf. subsection 2.4) and moreover $T_{b}=T_{t}=$ 
0 , but in order to trace back the place of each quantity in the formulae, the symbols are left as such. The mass fractions $c_{b}$ and $c_{t}$ in (31) and (32) are also considered as known constants.

The four constants $T_{i, r e f}, c_{i, r e f, l}, c_{i, r e f, g}$ and $J_{r e f}$ introduced above still need to be calculated in order to completely determine the reference solution. The equations they obey are derived from conditions (19) and (23)-(25) upon the substitution of (26)-(33) therein. One obtains the following system of four equations:

$$
\begin{aligned}
& -\left(T_{i, r e f}-T_{b}\right)+\lambda \frac{w_{g, r e f}}{\kappa} \frac{\left(T_{i, r e f}-T_{t}\right)}{1-e^{\left\{w_{g, r e f}(H-1) / \kappa\right\}}}=J_{r e f}, \\
& J_{\text {ref }}=-\frac{L e_{l}}{1-c_{i, r e f, l}}\left\{\left(c_{i, r e f, l}-c_{b}\right)+\psi_{s}\left(T_{i, r e f}-T_{b}\right)\right\}, \\
& 1=-\frac{(1-\rho)}{1-c_{i, r e f, g}} \frac{\left(c_{i, r e f, g}-c_{t}\right)}{\left.\left.1-e^{\left\{w_{g}, r e f\right.}(H-1) /(D L e) l\right)\right\}}, \\
& \frac{c_{i, r e f, g} \delta_{M}^{\prime \prime}}{1+c_{i, r e f, g}\left(\delta_{M}^{\prime \prime}-1\right)}=\frac{c_{i, r e f, l} \delta_{M}}{1+c_{i, r e f, l}\left(\delta_{M}-1\right)} \frac{K_{e}^{n}}{p_{i, r e f, g}} \text {. }
\end{aligned}
$$

This non-linear system (34)-(37) must be solved numerically for the four unknown constants, which results in a complete determination of the quasi-stationary reference solution. As illustrations, some of the reference temperature and mass fraction profiles are presented in Figs. 2 and 3 for the water-ethanol system with $c_{b}=0.1$ for several values of $H$. Typical numerical values of some parameters are also given in Table 3 . Let us also mention that a detailed analysis of the solutions obtained (which is actually not presented here) shows that the pressure does not change significantly across the system. Indeed, for values of $H$ between 1.5 to 101 , and for $p_{t}^{d}=1 \mathrm{~atm}$, it appears that $p_{i, r e f, g}^{d}-p_{t}^{d}$ is always smaller than $1 \mathrm{~Pa}$. Finally, note also that, as announced in section 2.3, equation (16) was not used to determine the solution given above. In case one is interested in the variation of $h$ with time, the above calculations must be repeated for $h \neq 1$ and (16) can then be used as a (non-linear) ODE for $h(t)$. Remark the weak deviations of the temperature with respect to $300 \mathrm{~K}$, in Fig. 2, which justifies our hypothesis to neglect the variations of the Henry constant (and, in principle, of other physical properties) with the temperature. Note from Fig. 3 that the mass fraction in the gas is an order of magnitude smaller than that in the liquid, which justifies the hypothesis to neglect the Soret effect in the gas phase. 


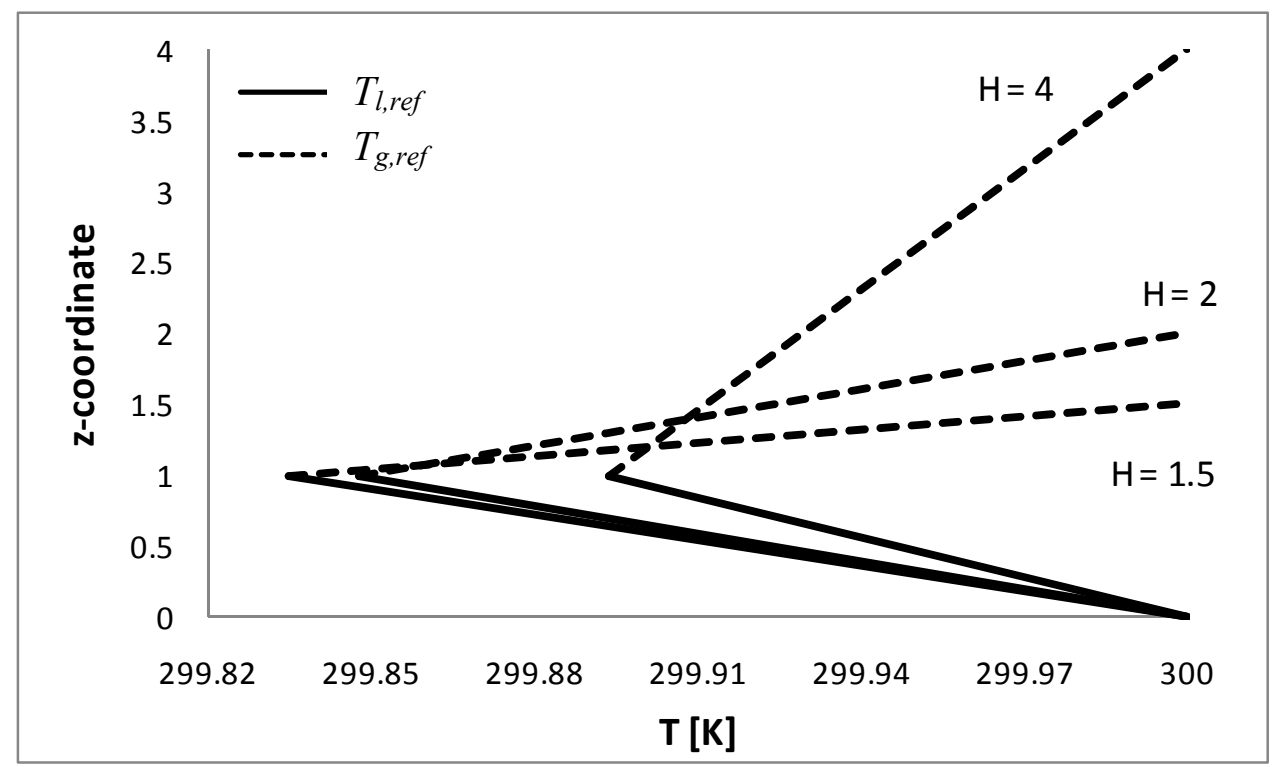

Fig. 2. Temperature distribution in the reference state (10 wt $\%$ ethanol in water)

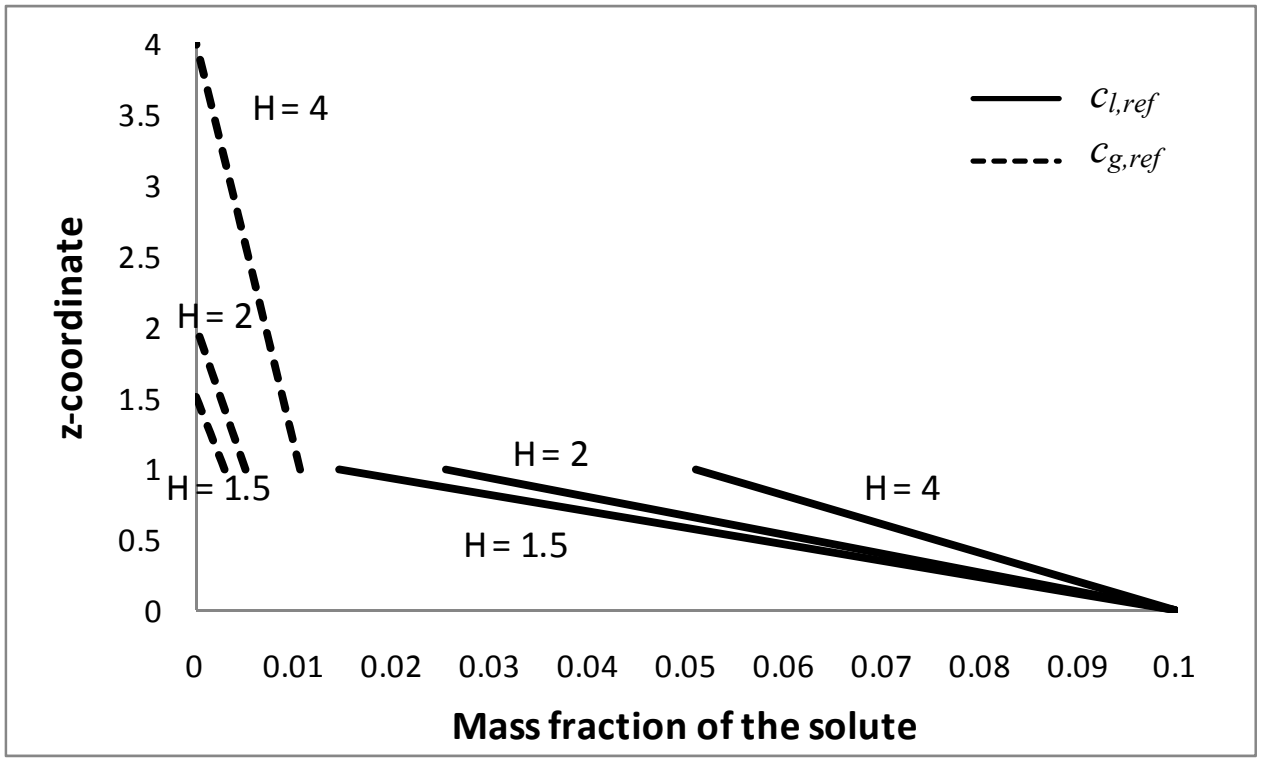

Fig. 3. Solute mass distribution in the reference state ( $10 \mathrm{wt} \%$ ethanol in water)

In the previous text, the quasi-stationary assumption has been used. It is interesting to investigate to what extent this assumption is valid (excluding very short times as mentioned in section 2.3). This can be done by examining the relevant time scales. For the liquid phase, the time scales in question are the time scale for the variation of the liquid height $\left(h^{d} /\left(-\dot{h}^{d}\right)\right)$, the diffusion time scale $\left(d_{l}^{2} / D_{l}\right)$, the thermal time scale $\left(d_{l}^{2} / \kappa_{l}\right)$ and the viscous time scale $\left(d_{l}^{2} / v_{l}\right)$. The first of them must be much larger than the other three for the quasi-stationary assumption to hold. The corresponding ratios give rise to the diffusion and thermal Péclet and Reynolds numbers that must be small. As typically $D_{l} \ll \kappa_{l}$ and $D_{l} \ll v_{l}$, it is the diffusion Péclet number in the liquid 
$P e_{l, \text { diff }}=\frac{d_{l}\left(-\dot{h}^{d}\right)}{D_{l}}=\frac{J_{r e f}}{L e_{l}}=\frac{\rho}{1-\rho} \frac{w_{g, r e f}}{L e_{l}}$

(with various equivalent expressions provided) that must be followed as the criterion of quasistationarity $\left(P e_{l, \text { diff }} \ll 1\right)$. Its values are shown in dimensionless form in Table 3 for a number of cases and turn out to be small indeed: the more so, the bigger $H$ is. Note that the concentration gradient in the liquid turns out to be of the order $O\left(P e_{l, d i f f}\right)$.

From the gas side, the corresponding criterion of quasi-stationarity is $d_{g}\left(-\dot{h}^{d}\right) / D_{g} \ll 1$. As $\rho \ll 1$, which brings about $\left|\dot{h}^{d}\right| \ll w_{g, r e f}$ (cf. (27) and (28)), the dimensionless number $d_{g}\left(-\dot{h}^{d}\right) /$ $D_{g}$ is anyway much smaller than what we shall call the Péclet number in the gas:

$P e_{g, \text { diff }}=d_{g} w_{g, r e f} / D_{g}$.

Thus, should it be that $P e_{g, d i f f} \ll 1$, then the quasi-stationarity criterion in the gas is satisfied all the more. On the other hand, $P e_{g, d i f f}$ is the criterion of non-linearity of the profiles (30) and (32) with respect to $z$. P $e_{g, \text { diff }}$ indeed happens to be small in our example, as substantiated by its values represented in Table 3, which explains why the profiles in the gas phase shown in Figs. 2 and 3 are almost linear, and at the same time justifies the quasi-stationary assumption Another interesting observation from Table 3 is that $P e_{g, \text { diff }}$ happens to be nearly equal to $\left(c_{i, r e f, g}-c_{t}\right)$, the two being small. Note that in the work [18], devoted to the Marangoni instability in an evaporating binary-liquid droplet, the same type of criteria have been elaborated to validate the quasi-stationary assumption. Their calculations showed as well that the gas phase could be treated as asymptotically steady and that the regression of the surface was negligible.

Table 3. Numerical values of some quantities in the reference solution for different values of $H$ (10 $\mathrm{wt} \%$ ethanol in water)

\begin{tabular}{lccccc}
\hline \hline \multicolumn{1}{c}{$H$} & $P e_{l, \text { diff }}$ & $P e_{g, \text { diff }}$ & $\left(T_{i, r e f}^{d}-T_{b}^{d}\right)[K]$ & $\left(c_{i, r e f, l}-c_{b}\right)$ & $\left(c_{i, r e f, g}-c_{t}\right)$ \\
\hline 1.5 & $8.66 * 10^{-2}$ & $2.99 * 10^{-3}$ & $-1.65^{*} 10^{-1}$ & $-8.54 * 10^{-2}$ & $2.98 * 10^{-3}$ \\
2 & $7.62 * 10^{-2}$ & $5.27 * 10^{-3}$ & $-1.52 * 10^{-1}$ & $-7.44 * 10^{-2}$ & $5.26 * 10^{-3}$ \\
4 & $5.14 * 10^{-2}$ & $1.07 * 10^{-2}$ & $-1.06 * 10^{-1}$ & $-4.88^{*} 10^{-2}$ & $1.06 * 10^{-2}$ \\
11 & $2.39 * 10^{-2}$ & $1.65 * 10^{-2}$ & $-4.98^{*} 10^{-2}$ & $-2.21 * 10^{-2}$ & $1.64 * 10^{-2}$ \\
101 & $3.02 * 10^{-3}$ & $2.09 * 10^{-2}$ & $-6.30^{*} 10^{-3}$ & $-2.74 * 10^{-3}$ & $2.07 * 10^{-2}$ \\
\hline \hline
\end{tabular}

Table 3 also shows that for increasing $H$, the mass fraction gradient in the liquid phase gets smaller and smaller $\left(c_{b}-c_{i, r e f, l} \ll c_{b}\right)$, which provides a justification for not considering in our model the variation of the material properties with the concentration in the liquid phase, even if some of them (for instance, the diffusion coefficient [24], the Soret coefficient (11) or $\gamma_{C}$ ) are rather sensitive to $c_{l}$. Note that one can also make use of the smallness of the Péclet numbers, and of some other effects, in order to build an approximate model of the stability problem, as we will show later in section 6 . Also provided in Table 3 are the numerical values of the temperature gradient for possible comparison by other readers. 


\section{Stability of the reference solution}

In order to study the stability of the reference solution, the time evolution of small perturbations with respect to this solution must be studied. These perturbations are introduced by expressing the unknowns as $T=T_{\text {ref }}+T^{\prime}, \quad \vec{v}=\vec{v}_{\text {ref }}+\vec{v}^{\prime}, \quad c=c_{\text {ref }}+c^{\prime}, \quad p=p_{\text {ref }}+p^{\prime}, \quad h=h_{\text {ref }}+h^{\prime}$. Following a standard procedure, these decompositions can be introduced in the bulk equations and boundary conditions, which are then linearized with respect to the infinitesimal perturbations. As far as the liquid-gas interface is concerned, it is important to stress that the corresponding boundary conditions must be expressed at $h=h_{r e f}+h^{\prime}$ and linearized with respect to $h$ '. Note that in the following the primes, denoting the perturbations of the reference state, will be omitted for simplicity.

The stability analysis is carried out in the framework of the so-called "frozen-time" approach, i.e. the evolution of perturbations is calculated in the form of normal modes superposed to each instantaneous snapshot of a time-evolving reference profile, as if the latter wasere stationary. On account of the quasi-stationarity assumption adopted in the present paper (see subsection 2.3), the frozen-time normal-mode approach tends to become exact: the smaller the Péclet numbers (see previous section), the more so. Of course, this tacitly implies that the perturbations evolve on the time scale of diffusion in the liquid at the slowest, which will actually be the case for all the modes but one considered hereafter. This exceptional mode will be a slow one, associated with the time evolution of the liquid layer thickness, for which the frozen-time approach bears all its "standard" shortcomings. Under the undeformable surface assumption used here throughout, it will be just an isolated mode corresponding to a zero wavenumber. For the sake of concreteness, we shall treat it here assuming that it is only the thickness of the liquid layer that is perturbed, and not the total thickness of the two layers (i.e. the perturbation of $H$ is equal to zero), even though other arrangements are in principle possible within the conceptual framework set forth in the beginning of section 2 .

The horizontal components of the velocity can be eliminated from the equations as usual (by applying $\nabla \times \nabla \times$ to the momentum equation). The normal modes are introduced as follows:

$$
\begin{aligned}
& \left(\begin{array}{c}
w_{l} \\
T_{l} \\
c_{l} \\
p_{l}
\end{array}\right)=e^{\left\{\sigma t+i\left(k_{x} x+k_{y} y\right)\right\}}\left(\begin{array}{c}
W_{l}(z) \\
\theta_{l}(z) \\
C_{l}(z) \\
P_{l}(z)
\end{array}\right), \\
& \left(\begin{array}{c}
w_{g} \\
T_{g} \\
c_{g} \\
p_{g}
\end{array}\right)=e^{\left\{\sigma t+i\left(k_{x} x+k_{y} y\right)\right\}}\left(\begin{array}{c}
W_{g}(z) \\
\theta_{g}(z) \\
C_{g}(z) \\
P_{g}(z)
\end{array}\right), \\
& h=e^{\left\{\sigma t+i\left(k_{x} x+k_{y} y\right)\right\}} h_{0}, \text { with } h_{0}=0 \text { if } \vec{k} \neq 0 .
\end{aligned}
$$


In (38)-(40), $\sigma$ is the complex growth rate of the perturbations and $\vec{k}=\left(k_{x}, k_{y}\right)$ is the wavevector (with the wavenumber $k \equiv \sqrt{k_{x}^{2}+k_{y}^{2}}$ ), whereas $W, \theta, C$ and $P$ are the complex amplitudes (functions of $z$ ). In (40), the vanishing of $h_{0}$ for non-zero wave vectors is a consequence of the assumption of an undeformable liquid-gas interface. Note that $h_{0}$ does not depend on $z$, of course.

The (linear) equations for the amplitude of the perturbations, as derived from (3)-(10) and valid for both zero and non-zero wavenumber, are found to be

$-P r_{l}{ }^{-1} \sigma\left(D_{z}^{2}-k^{2}\right) W_{l}+\left(D_{z}^{2}-k^{2}\right)^{2} W_{l}=R a_{l} k^{2} \theta_{l}+R s_{l} L e_{l} k^{2} C_{l}$,

$-\sigma \theta_{l}+\left(D_{z}^{2}-k^{2}\right) \theta_{l}=W_{l}\left(T_{i, r e f}-T_{b}\right)$,

$-\sigma C_{l}+L e_{l}\left(D_{z}^{2}-k^{2}\right) C_{l}=W_{l}\left(c_{i, r e f, l}-c_{b}\right)-L e_{l} \psi_{s}\left(D_{z}{ }^{2}-k^{2}\right) \theta_{l}$,

$-P r_{l}{ }^{-1}\left(\sigma+w_{g, r e f} D_{z}\right)\left(D_{z}^{2}-k^{2}\right) W_{g}+v\left(D_{z}^{2}-k^{2}\right)^{2} W_{g}=\alpha R a_{l} k^{2} \theta_{g}+\varepsilon R s_{l} L e_{l} k^{2} C_{g}$,

$-\left(\sigma+w_{g, r e f} D_{z}\right) \theta_{g}+\kappa\left(D_{z}{ }^{2}-k^{2}\right) \theta_{g}=W_{g} D_{z} T_{g, r e f}$,

$-\left(\sigma+w_{g, r e f} D_{z}\right) C_{g}+D L e_{l}\left(D_{z}^{2}-k^{2}\right) C_{g}=W_{g} D_{z} c_{g, r e f}$.

Later on, we will eventually need the following expression for the pressure amplitude in the gas phase, which is easily deduced starting from the perturbed form of (7) and the horizontal component of (8):

$P_{g}=-\frac{P r_{l}^{-1} \rho}{k^{2}}\left(\sigma+w_{g, r e f} D_{z}\right) D_{z} W_{g}+\frac{\mu}{k^{2}}\left(D_{z}^{2}-k^{2}\right) D_{z} W_{g}$.

The boundary conditions are derived from (13), (14), (15)-(19) and (21)-(25). At $z=0$, one obtains

$W_{l}=0, \quad D_{z} W_{l}=0, \quad \theta_{l}=0, \quad C_{l}=0$.

At $z=H$, the boundary conditions for the amplitudes of the perturbations write

$\theta_{g}=0, \quad C_{g}=0, \quad D_{z}^{2} W_{g}+k^{2} W_{g}=0, \quad-P_{g}+2 \mu D_{z} W_{g}=0$.

As said above, the conditions at the moving interface $z=1+h$ are linearized with respect to $h$ and with respect to all perturbations. One obtains the following relations at $z=1$ :

$\theta_{l}+D_{z} T_{l, r e f} h_{0}=\theta_{g}+D_{z} T_{g, r e f} h_{0}$

$-D_{z} \theta_{l}-D_{z}^{2} T_{l, r e f} h_{0}+\lambda D_{z} \theta_{g}+\lambda D_{z}^{2} T_{g, r e f} h_{0}=\frac{\rho}{1-\rho}\left(W_{g}-W_{l}\right)$,

$D_{z} W_{l}=D_{z} W_{g}, \quad\left(W_{l}-\sigma h_{0}\right)=\rho\left(W_{g}-\sigma h_{0}\right)$,

$-\mu\left(D_{z}^{2}+k^{2}\right) W_{g}+\left(D_{z}^{2}+k^{2}\right) W_{l}+M a k^{2} \theta_{l}+M s L e_{l} k^{2} C_{l}=0$,

$\frac{\rho}{1-\rho}\left(W_{g}-W_{l}\right)=-\frac{L e_{l}}{1-c_{l, r e f}}\left[\left(D_{z} C_{l}+D_{z}^{2} c_{l, r e f} h_{0}\right)+\psi_{S}\left(D_{z} \theta_{l}+D_{z}^{2} T_{l, r e f} h_{0}\right)\right]+\frac{J_{r e f}}{1-c_{l, r e f}}\left(C_{l}+D_{z} c_{l, r e f} h_{0}\right)$,

$\frac{\rho}{1-\rho}\left(W_{g}-W_{l}\right)=-\frac{\rho D L e_{l}}{1-c_{g, r e f}}\left(D_{z} C_{g}+D_{z}^{2} c_{g, r e f} h_{0}\right)+\frac{J_{r e f}}{1-c_{g, r e f}}\left(C_{g}+D_{z} c_{g, r e f} h_{0}\right)$, 
$\frac{\delta_{M}^{\prime \prime} p_{i, r e f, g}}{\left[1+c_{g, r e f}\left(\delta_{M}^{\prime \prime}-1\right)\right]^{2}}\left(C_{g}+D_{z} c_{g, r e f} h_{0}\right)+\frac{\delta_{M}^{\prime \prime} c_{i, r e f, g}}{1+c_{g, r e f}\left(\delta_{M}^{\prime \prime}-1\right)}\left(P_{g}+D_{z} p_{g, r e f} h_{0}\right)=\frac{\delta_{M} K_{e}^{n}}{\left[1+c_{l, r e f}\left(\delta_{M}-1\right)\right]^{2}}\left(C_{l}+\right.$

$\left.D_{z} c_{l, r e f} h_{0}\right)$,

with $P_{g}$ given by (47) and with $h_{0}=0$ if $\vec{k} \neq 0$. Here we have also taken into account (26) and that $D_{z} w_{g, r e f}=0$ in accordance with (27).

Thus, an eigenvalue problem for the growth rate of perturbations has been obtained, the results for which are analyzed below.

\section{Linear stability results}

\subsection{Numerical results for the non-zero mode case}

In the case $\vec{k} \neq 0$, the eigenvalue problem presented above is solved using a Tau-Chebyshev method, which is rather classical and thus not recalled here (see for instance $[25,26]$ ). Before proceeding to the results, let us stress that in the classical studies of the Rayleigh-Marangoni problems, the control parameter is just the imposed temperature gradient. In the present case of evaporation, we assume $T_{b}^{d}=T_{t}^{d}$ so that the resulting temperature gradients are a consequence of the evaporation process, which is controlled by $c_{t}$ above the gas not being in equilibrium (in the sense of Henry's law) with $c_{b}$ at the bottom of the liquid. Here, we shall take $c_{t}=0$ and study two different values of $c_{b}$. With these boundary conditions and with clearly defined components in the liquid and gas phases (water, ethanol and air), the main control parameters we are left with are just the thicknesses of the liquid and gas layers, $d_{l}$ and $d_{g}$. In principle, the background temperature $T_{b}^{d}=T_{t}^{d}$ also plays a role as the material properties (and perhaps most notably the Henry coefficient) depend on it. An imposed pressure could also be used to control the mass fraction in the gas phase (and influence the mass fraction in the liquid phase by Henry's law). However, the two latter types of control are not considered in the present paper. The control parameters $\left(d_{l}\right.$ and $\left.d_{g}\right)$ enter into the dimensionless formulation by means of the dimensionless numbers $G a_{l}, R a_{l}, R s_{l}, M a_{l}, M s_{l}$ and $H$. Varying $d_{l}$ and $d_{g}$ means varying these numbers, albeit not independently once the system and the constraints have been fixed physico-chemically.

The marginal stability curves are obtained by calculating, as a function of the wavenumber $k$, the value of the liquid layer thickness $d_{l}$ for which the condition $\operatorname{Re}(\sigma)=0$ (vanishing of the largest real part of all growth rates) holds, keeping all the other parameters fixed. First let us mention that the instability is always monotonic, since our numerical results show that $\operatorname{Im}(\sigma)$ is always equal to 0 for the marginal states. Fig. 4(a) presents the neutral curves corresponding to $H=2$, 11 and 101. Fig. 4(b) is a plot of the critical wavenumber as a function of $H$ and shows that this critical wavenumber remains approximately constant and close to 2 over the whole range of values of $H$. 
(a)

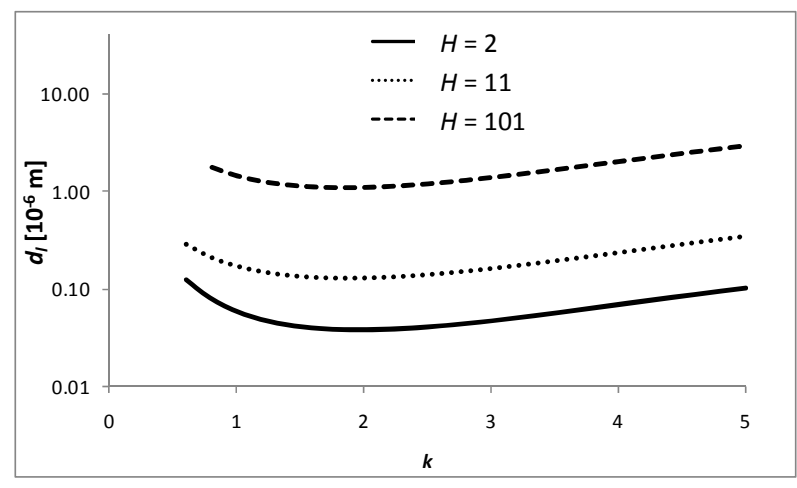

(b)

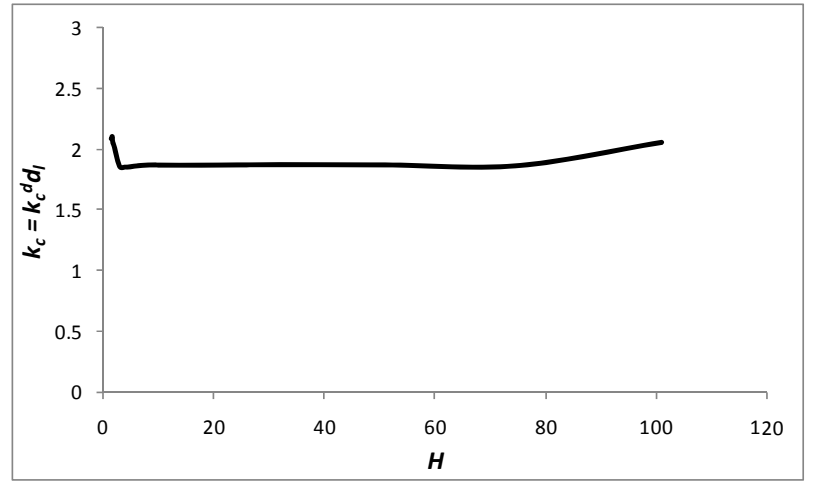

Fig. 4. Neutral stability curve in terms of the liquid layer thickness for $H=2,11$ and 101 (10 $\mathrm{wt} \%$ ethanol in water)

To examine the influence of the gas layer thickness $\left(d_{g}\right)$ on the stability threshold, we have plotted in Fig. 5 the critical liquid thickness (i.e. the minimum of the neutral stability curve of Fig. 4) as a function of $H$, and also as a function of $d_{g}$. Figs. 5(a) and 5(b) correspond to $c_{b}=0.1$. In Fig. 5(c) and (d), the results for $c_{b}=0.001$ are also shown for comparison.

(a)

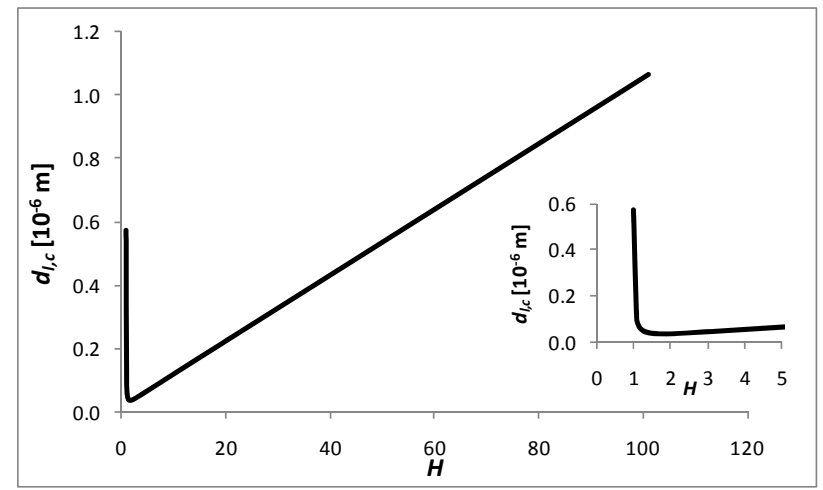

(c)

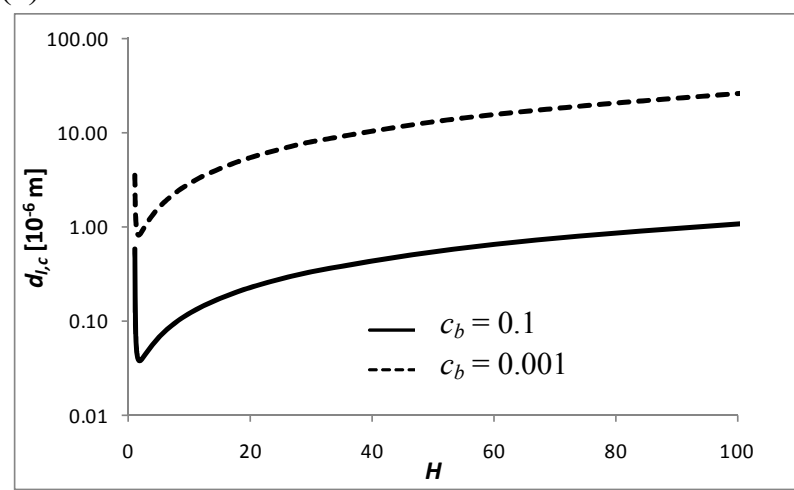

(b)

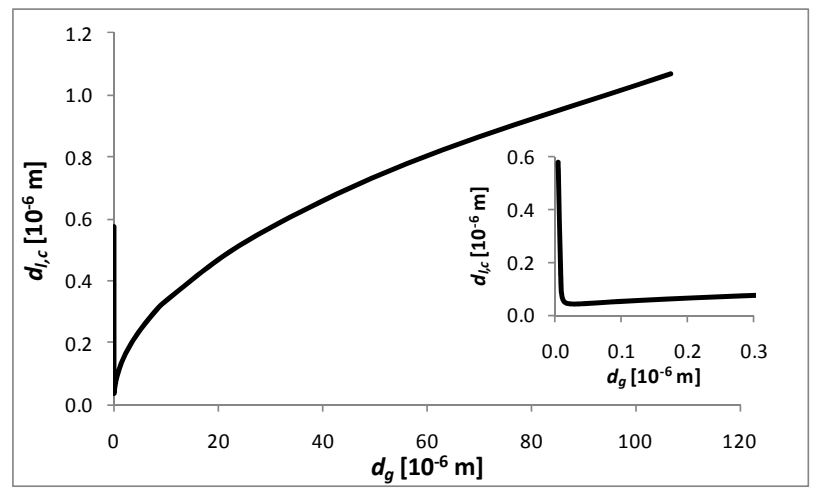

(d)

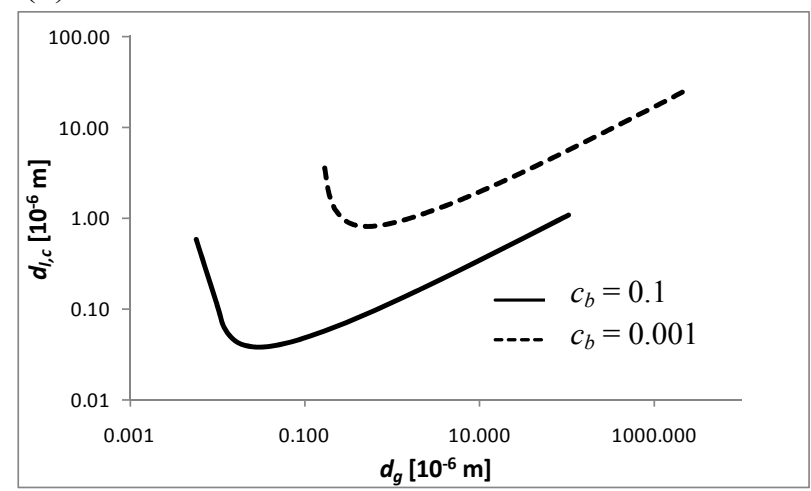

Fig. 5. The critical liquid thickness as a function of $H(\mathrm{a})$ and $d_{g}(\mathrm{~b})$ for an ethanol/water system with $c_{b}=0.1$ (10 wt\% ethanol in water), and the same, albeit in different scales, together with the results for $c_{b}=0.001(0.1 \mathrm{wt} \%$ ethanol in water $)(\mathrm{c}, \mathrm{d})$ 
Fig. 5(a), corresponding to $c_{b}=0.1$, shows that there is a linear relation between the critical thickness and $H$ for sufficiently large values of $H$ (practically, higher than 2). This corresponds to a scaling $d_{l, c} \sim d_{g}^{0.5}$ for large $d_{g}$, which is indeed observed in Fig. 5(b). Note also that for very thin gas layers (Fig. 5(b)), the critical liquid height increases very sharply and diverges to infinity, leaving a certain minimum in between. A physical interpretation of this minimum will be presented in section 6, in relation with Fig. 12. It can also be noted that the critical liquid heights are rather small. This means that liquid layers of reasonable thicknesses will be very unstable. This somehow confirms the scaling analysis performed in [16]. Note that the critical wavenumber for these results always appeared to be around 2. For $c_{b}=0.001$ (Figs. 5(c) and (d)), the same observations are made and the same trends are observed. The only difference is that the critical liquid thickness is roughly 25 times larger for $c_{b}=0.001$ than for $c_{b}=0.1$. The interest of using two different bottom mass fractions is to find out whether a very dilute liquid produces different results. It is clearly shown that the decrease of the solute concentration has a stabilizing effect. One should remember, however, that the results for $c_{b}=0.001$ are obtained while neglecting the evaporation of water (which is a strong limitation for so small concentrations).

\subsection{Numerical results for the zero wavenumber case}

The solution of the eigenvalue problem for $\vec{k}=0$ does not require the use of the Tau-Chebyshev method. First, a closed form solution can be obtained easily for the perturbations $W, \theta$ and $C$ [27] leaving some unknown constants (including the growth rate and the amplitude $h_{0}$ of the liquid thickness perturbation). Then these unknown constants can be numerically determined by solving the non-linear algebraic equations provided by the boundary conditions (50), (51), the second condition (52), (54)-(56) and a normalization condition for the solution. In particular, an infinite numbers of growth rates can be numerically determined for any fixed value of $H$. In the zero-mode case, these growth rates do not depend on $M a$ and $M s$ and depend on the dimensionless numbers $R a_{l}$ and $R s_{l}$ only via the reference pressure in the gas phase. Here, though, this dependence is so weak $\left(p_{i, r e f, g}-p_{t}<1 \mathrm{~Pa}\right)$ that it can be neglected. Therefore, the growth rates depend significantly on the control parameters $\left(d_{l}\right.$ and $\left.d_{g}\right)$ only via the value of $H$. The results of these calculations can be summarized as follows. For any given $H$, all the calculated growth rates have negative real parts, except one which is real and positive. The latter is not unexpected and indicates that the evaporation rate increases with the decrease of the liquid layer thickness, which within the frozen-time approach boils down to this positive growth rate.

Note also that this positive value appears to be much smaller than the absolute value of the real

parts of all other values of the growth rate. Since $\sigma=\frac{\dot{h}}{h}$ (note the different meaning of this quantity as compared to the one used in section 3 , since in this case it concerns the perturbations and not the reference state), the smallness of the positive growth rate expresses once again that 
the characteristic time of the variation of the depth of the liquid is much longer than the other time scales in the problem.

As a consequence of the above discussion, we can conclude that no mode with $\vec{k}=0$ is able to destabilize the reference state unless on a very long time scale, comparable to the time it takes to fully evaporate the layer. In principle, all what concerns this mode would be best captured by relaxing the limitation of surface non-deformability used in the present paper and developing a lubrication-approximation (non-linear) theory of an evaporating liquid layer much along the lines of [28] (where a similar homogeneous mode has been identified). In the present paper, however, we shall rather be interested in an evaporation-induced Bénard convection appearing on a shorter time scale, whose existence is indicated by the linear stability analysis results of subsection 5.1.

\subsection{Comparison of the solutal, thermal and Soret contributions to the Rayleigh and Marangoni} effects

In the following subsections 5.3-5.6, we analyze in more detail the physical mechanisms responsible for the instability and we start here by comparing the thermal and solutal contributions, for both the Rayleigh and the Marangoni effects.

Consider first the Rayleigh effect and let us combine equations (41)-(43) for marginal perturbations $\sigma=0$ to deduce the following single equation for $W_{l}$ only:

$\left(D_{z}{ }^{2}-k^{2}\right)^{3} W_{l}=-k^{2}\left\{R a_{l}\left(T_{b}-T_{i, r e f}\right)+R s_{l}\left[\left(c_{b}-c_{i, r e f, l}\right)-L e_{l} \psi_{S}\left(T_{b}-T_{i, r e f}\right)\right]\right\} W_{l}$.

Three additive terms depending on gravity can be distinguished in (57). Accordingly, three contributions to the Rayleigh effects can then be identified and quantified. Two of them describe thermal and solutal effects by the following dimensionless numbers:

$R a^{*}=R a_{l}\left(T_{b}-T_{i, r e f}\right)=\frac{\alpha_{l} g d_{l}^{3}\left(T_{b}^{d}-T_{i, r e f}^{d}\right)}{\kappa_{l} v_{1}}$,
$R s^{*}=R s_{l}\left(c_{b}-c_{i, r e f, l}\right)=\frac{\varepsilon_{l} g d_{l}^{3}\left(c_{b}-c_{i, r e f, l}\right)}{D_{l} v_{1}}$,

The Soret effect is then described by the following dimensionless number:

$R_{\Psi}^{*}=R s_{l} L e_{l} \psi_{S}\left(T_{b}-T_{i, r e f}\right)=\frac{\varepsilon_{l} g d_{l}^{3}\left(T_{b}^{d}-T_{i, r e f}^{d}\right)}{\kappa_{l} \nu_{l}} S_{l, 0}$.

$R a^{*}$ can be defined as a "true" thermal Rayleigh number and accounts for the thermal Rayleigh effect. $R s^{*}$ is the "true" solutal Rayleigh number and accounts for the solutal Rayleigh effect. The $R_{\Psi}^{*}$ number is referred to as the "Soret Rayleigh number". The latter number stands for the density effects that are solutal in nature $\left(\varepsilon_{l}\right)$ but caused by thermodiffusion (Soret effect). In order to assess the relative importance of these effects with respect to one another, three ratios can be defined: the solutal and thermal effects can be compared by examining the number $R s^{*} / R a^{*}$, while the solutal and the Soret-Rayleigh effects are compared by considering the number $R s^{*} / R_{\Psi}^{*}$ and the thermal effect and the Soret-Rayleigh effect can be compared using the number $R a^{*} / R_{\Psi}^{*}$. 
The same evaluation can be done for the Marangoni effect, but unlike the Rayleigh case one can no longer derive a single relation where one could observe the relative importance of various contributions as clearly as in (57). However, a straightforward order-of-magnitude argument allows introducing "true" Marangoni numbers, whose definitions fully parallel those given for the Rayleigh numbers. For the thermal and solutal effects, this gives

$M a^{*}=M a\left(T_{b}-T_{i, r e f}\right)=\frac{\gamma_{T} d_{l}\left(T_{b}^{d}-T_{i, r e f}^{d}\right)}{\kappa_{l} \mu_{1}}$,

$M s^{*}=M s\left(c_{b}-c_{i, r e f, l}\right)=\frac{\gamma_{C} d_{l}\left(c_{b}-c_{i, r e f, l}\right)}{D_{l} \mu_{l}}$,

For the Soret effect, this gives

$M_{\Psi}^{*}=M s L e_{l} \psi_{S}\left(T_{b}-T_{i, r e f}\right)=\frac{\gamma_{C} d_{l}\left(T_{b}^{d}-T_{i, r e f}^{d}\right)}{\kappa_{l} \mu_{l}} S_{l, 0}$.

$M a^{*}$ is the "true" thermal Marangoni number, with $M s^{*}$ being the "true" solutal Marangoni number, while $M_{\Psi}^{*}$ being referred to as the "Soret Marangoni number". This number stands for the surface tension effects that are solutal in nature $\left(\gamma_{C}\right)$ but caused by thermodiffusion. In the same manner as in the Rayleigh case, the comparison between the solutal effect and the thermal and Soret-Marangoni effects can be performed by considering the ratios $M s^{*} / M a^{*}, M s^{*} / M_{\Psi}^{*}$ and $M a^{*} / M_{\Psi}^{*}$. After defining all these ratios, one can first note that in fact two of them are equal, with $\frac{R s^{*}}{R_{\Psi}^{*}}=\frac{M s^{*}}{M_{\Psi}^{*}}=\frac{\left(c_{b}-c_{i, r e f, l}\right)}{\left(T_{b}^{d}-T_{i, r e f}^{d}\right) L e_{l} S_{l, 0}}$.

This number, calculated for a liquid thickness equal to its critical value (function of $d_{g}$ ), represents the relative importance of the solutal and Soret effects for both the Rayleigh and Marangoni cases. In Fig. 6, it is plotted as a function of the gas layer thickness $d_{g}$ (for the waterethanol system, with $c_{b}=0.1$ ).

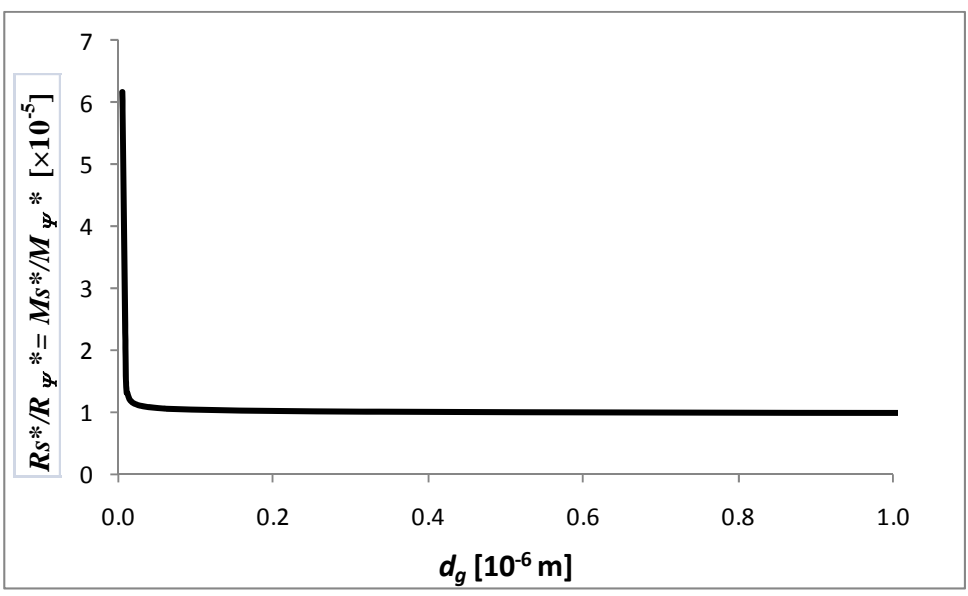

Fig. 6. The ratios of the solutal and the Soret contributions as a function of $d_{g}(10 \mathrm{wt} \%$ ethanol in water)

On the other hand, the ratios of the thermal and the Soret contributions are given by: $\frac{R a^{*}}{R_{\Psi}^{*}}=\frac{\alpha_{l}}{\varepsilon_{l} S_{l, 0}} \approx 3.82$, 
$\frac{M a^{*}}{M_{\Psi}^{*}}=\frac{\gamma_{T}}{\gamma_{C} S_{l, 0}} \approx 1.78$,

where the estimations are made for the water-ethanol system $\left(c_{b}=0.1\right)$ and show that the two effects are of the same order of magnitude, although the thermal one is somewhat stronger (especially in the Rayleigh case).

Fig. 6 together with (62) show that the solutal contribution is by far the biggest for both the Rayleigh and the Marangoni effects. This can be summed up by the following symbolic statement: Soret $\lesssim$ thermal « solutal. Note that for the case $c_{b}=0.001$, this statement turns out to remain valid.

\subsection{Marangoni versus Rayleigh effects}

The relative importance of the Rayleigh and the Marangoni effects can be evaluated as follows. First, the values of the thermal and solutal Rayleigh numbers are calculated at the critical condition, considering no Marangoni effect (setting artificially $M s \equiv 0$ and $M a \equiv 0$ ). Second, the values of the thermal and solutal Marangoni numbers are calculated at the critical condition, considering no Rayleigh effect (setting artificially $R s \equiv 0$ and $R a \equiv 0$ ). The results are shown in Table 4 for a number of $H$ values. Table 4 also shows the values for the thermal/solutal Rayleigh/Marangoni numbers when all the effects are taken into account.

Table 4. Values of the "true" Rayleigh and Marangoni numbers, equations (58)-(61), at the critical condition ( $10 \mathrm{wt} \%$ ethanol in water)

\begin{tabular}{|c|c|c|c|c|c|c|}
\hline \multirow[b]{2}{*}{$H$} & \multicolumn{3}{|c|}{ Only Rayleigh effect is considered } & \multicolumn{3}{|c|}{ Only Marangoni effect is considered } \\
\hline & $R a^{*}$ & $R s^{*}$ & $d_{l}[\mathrm{~m}]$ & $M a^{*}$ & $M s^{*}$ & $d_{l}[\mathrm{~m}]$ \\
\hline 2 & $3.45^{*} 10^{-2}$ & $9.63 * 10^{2}$ & $2.26^{*} 10^{-4}$ & $5.12^{*} * 10^{-3}$ & $3.06 * 10^{2}$ & $3.83 * 10^{-8}$ \\
\hline 11 & $3.81 * 10^{-2}$ & $9.66 * 10^{2}$ & $3.39 * 10^{-4}$ & $5.70 * 10^{-3}$ & $3.09 * 10^{2}$ & $1.30 * 10^{-7}$ \\
\hline \multirow[t]{2}{*}{101} & $3.64 * 10^{-2}$ & $9.03 * 10^{2}$ & $6.64 * 10^{-4}$ & $5.93 * 10^{-3}$ & $3.15 * 10^{2}$ & $1.07 * 10^{-6}$ \\
\hline & \multicolumn{3}{|c|}{ All effects are considered } & & & \\
\hline$H$ & $R a^{*}$ & $R s^{*}$ & & $M a^{*}$ & $M s^{*}$ & $d_{l}[\mathrm{~m}]$ \\
\hline 2 & $1.68 * 10^{-13}$ & $4.71 * 10^{-9}$ & & $5.12^{*} 10^{-3}$ & $3.06 * 10^{2}$ & $3.83 * 10^{-8}$ \\
\hline 11 & $2.18^{*} 10^{-12}$ & $5.51 * 10^{-8}$ & & $5.70^{*} 10^{-3}$ & $3.09 * 10^{2}$ & $1.30 * 10^{-7}$ \\
\hline 101 & $1.51 * 10^{-10}$ & $3.75 * 10^{-6}$ & & $5.91 * 10^{-3}$ & $3.14 * 10^{2}$ & $1.07 * 10^{-6}$ \\
\hline
\end{tabular}

It is clearly seen that the Marangoni effect is much stronger than the Rayleigh effect at the instability threshold, i.e. the instability is primarily due to the former. This is not surprising given the very small critical thicknesses obtained here, for which the surface effects must definitely dominate over the bulk ones.

\subsection{Which effect is the most important?}

From the results presented in subsections 5.3 and 5.4 it can be concluded that at the critical conditions, the solutal effect is much stronger than the thermal effect and that the Marangoni effect is much stronger than the Rayleigh effect. This suggests that it is only the solutal 
Marangoni effect that is primarily responsible for the instability in question, even when all the effects are taken into consideration. The same conclusion is arrived at in [16] in the case of evaporation of a dilute polymer solution. It should be noted, though, that in [16] it is the solvent that evaporates. Still, in this case too, the evaporation effectively increases the surface tension, hence it is essentially the same mechanism as studied here. Unfortunately, in [18], such a comparison of the mutual importance of the thermal and the solutal Marangoni effects for the particular system under consideration there (20\% heptane and $80 \%$ hexadecane) does not seem to be considered, the analysis being rather in the form of a parametric study formally involving both $M a$ and $M s$.

To illustrate the solutal Marangoni nature of the instability in this work, a calculation is performed neglecting the Soret, $R a, R s$ and $M a$ effects and keeping only the $M s$ effect. The results are presented in Fig. 7 for $H=2$ and 101, which confirms the above conclusion. The same holds for the case $c_{b}=0.001$. Therefore, in what follows, it is in terms of the solutal Marangoni number only that it seems to be most appropriate to represent the marginal conditions.

(a)

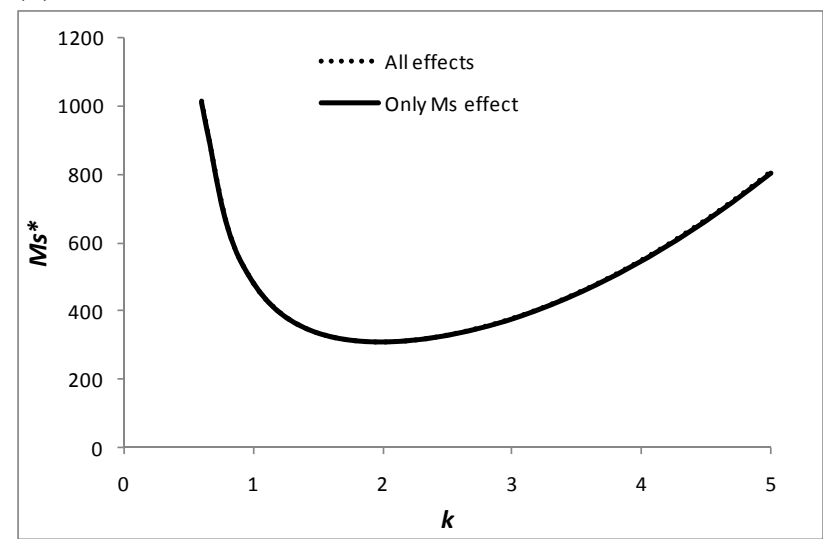

(b)

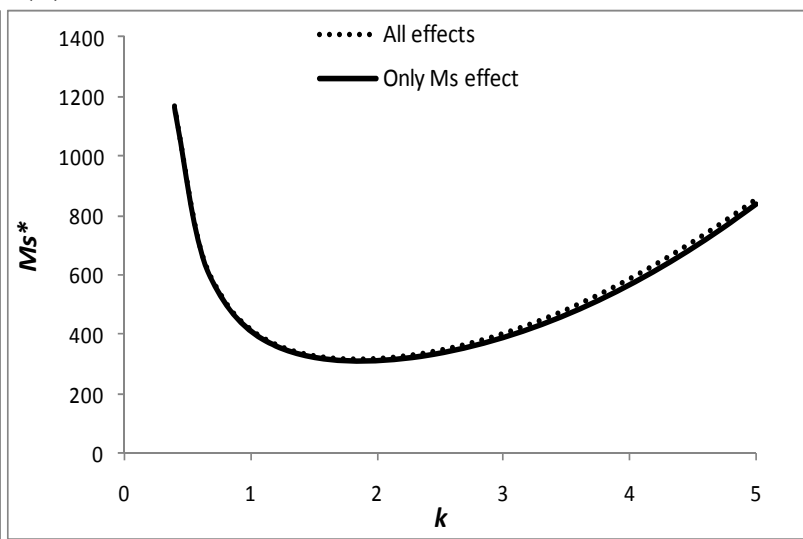

Fig. 7. The marginal stability curve in terms of the "true" solutal Marangoni number comparing the full analysis with the consideration of the solutal Marangoni effect only for $H=2$ (a) and $H=$ 101 (b) in the case of an ethanol/water mixture of 10/90 wt $\%$

\subsection{Marginal stability curves in terms of the solutal Marangoni number}

The neutral stability curves, already considered in subsection 5.1, can also be represented in terms of the solutal Marangoni number. This is presented in Fig. 8, for different values of $H$. 


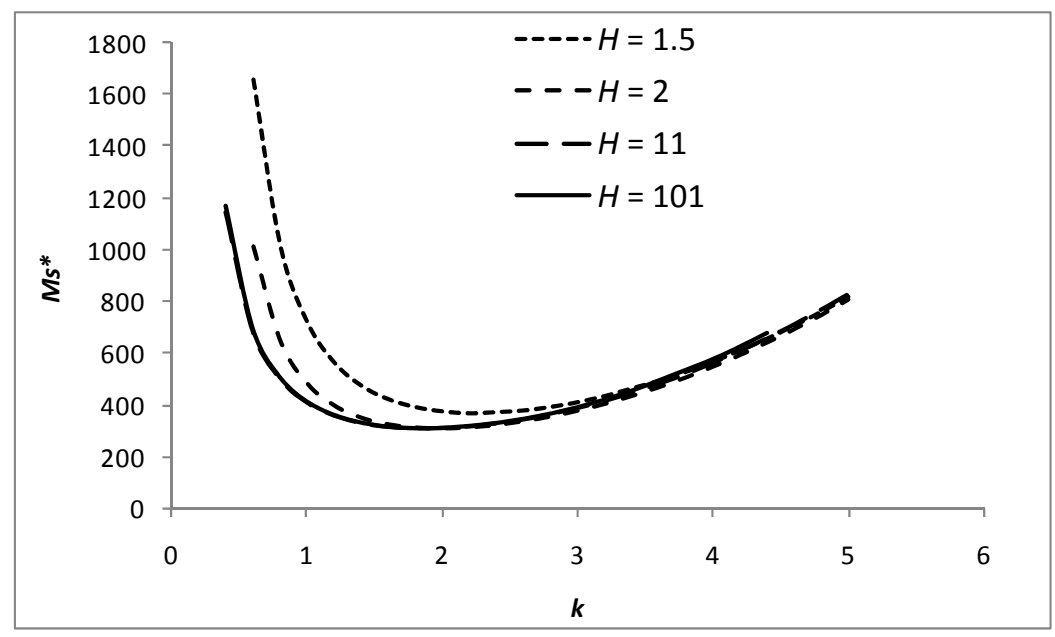

Fig. 8. The "true" solutal Marangoni number as a function of the wavenumber at the marginal condition for different values of $H$ in the case of an ethanol/water mixture of $10 / 90 \mathrm{wt} \%$

We see that the form of the curve in terms of the "true" solutal Marangoni number $M s^{*}$, equation (61), is not too much affected by the value of $H$ for large wavenumbers. It is not quite so for smaller wavenumbers, especially for low $H$ values. The physical reason is that for large $k$, the typical length scale of the perturbation pattern is small, so that the perturbations do not "feel" the top boundary, and hence the dependence on $H$ tends to disappear. In the opposite limit (of small $k$ ), this is obviously not the case, and the dependence on $H$ becomes well pronounced. This result will also be confirmed in section 6.2 on the basis of an approximate model. Fig. 9 shows the critical values of $M s^{*}$ as a function of the gas layer thickness.

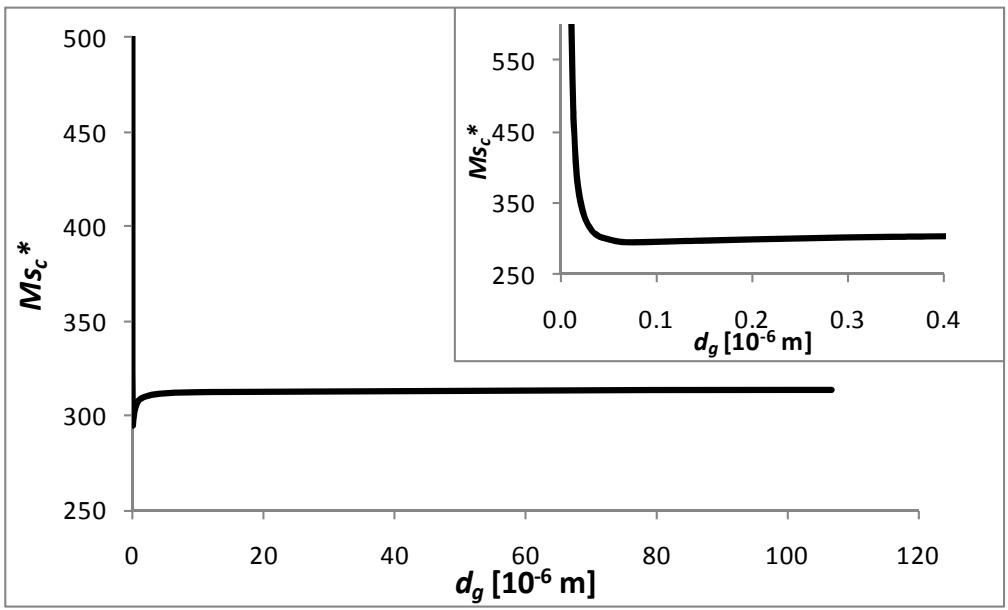

Fig. 9. The critical solutal Marangoni number $M s^{*}$ as a function of the gas layer thickness for an ethanol/water mixture of 10/90 wt \%

For small $d_{g}$, as $d_{g}$ is decreased, the critical $M s *$ is seen to behave non-monotonically. It turns out that this small- $d_{g}$ behavior is heavily dependent upon the hydrodynamic boundary conditions imposed at the top of the gas layer (cf. subsection 5.7). On the contrary, for larger $d_{g}$, such 
dependence is minimal, because the hydrodynamic top boundary conditions loose their importance as the gas layer thickness is increased.

\subsection{Influence of the top boundary condition for the gas velocity}

The hydrodynamic top boundary conditions in the gas phase that have been used until now are to a certain extent heuristic. It is therefore of interest to investigate how the results change if a different kind of boundary conditions is imposed instead. Having already tried two "soft" conditions (constant normal stress and zero tangential stress), we now examine two more "restrictive" alternatives: the no-slip and a fixed vertical velocity at the top boundary. In terms of the perturbations, the latter are given respectively by

$D_{z} W_{g}=0$,

and

$W_{g}=0$

at $z=H$.

The no-slip and fixed normal velocity conditions are combined one by one with the conditions for the normal and tangential stresses, forming four different combinations for which the results are presented in Fig. 10. Otherwise, the stability analysis is performed as developed earlier in this paper.

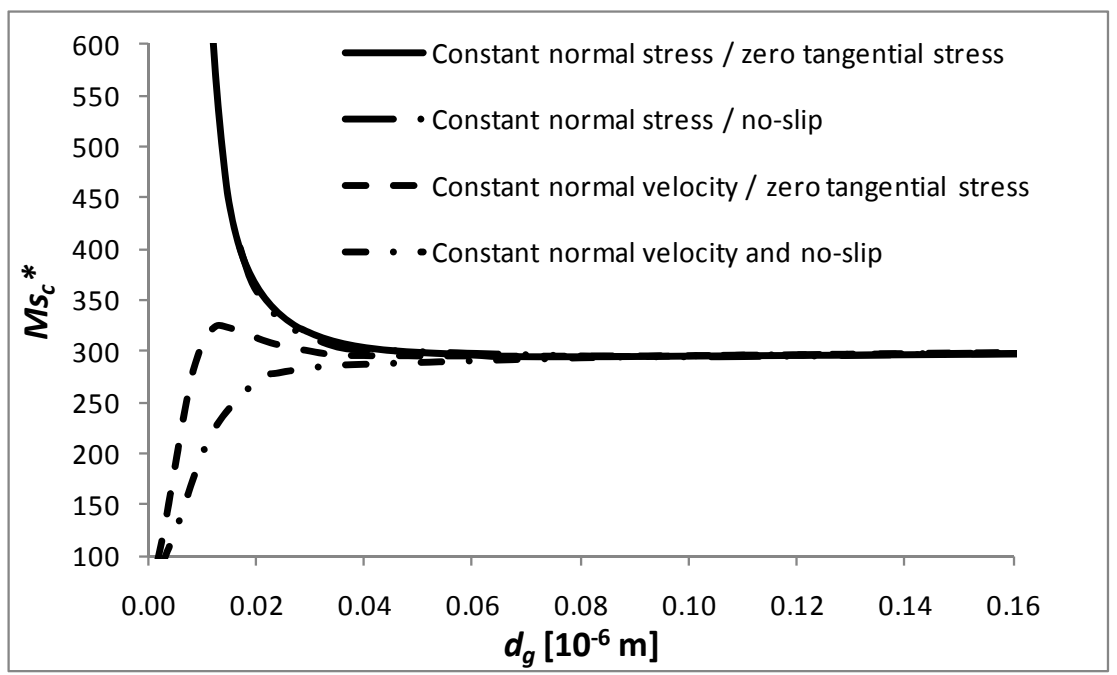

Fig. 10. The critical solutal Marangoni number as a function of the gas layer thickness for different kinds of hydrodynamic boundary conditions at the top boundary ( $10 \mathrm{wt} \%$ ethanol in water)

It turns out that the combination "constant normal stress / no-slip" shows no significant difference with respect to the originally used combination "constant normal stress / zero tangential stress". This means that it is first of all the "constant normal stress" and the "constant normal velocity" conditions that make the difference for sufficiently small $d_{g}$, the latter being even able to induce a peculiar instability, with a critical solutal Marangoni number that decreases 
for smaller and smaller $d_{g}$. To our knowledge, this peculiar instability is not discussed in the literature, and we believe that it is caused by the evaporation-induced convective transport in the gas, acting similarly to a "wind" exerting some viscous stress back on the liquid layer. At large $d_{g}$, with no restriction on the normal velocity at the top boundary, this "wind" is less constrainted, and moves away without influencing significantly the instability in the liquid. However, at small $d_{g}$ and with a constant normal velocity at the top boundary, this "wind" gets more restrained, hence increasing the viscous feedback on the liquid layer. For gas layer thicknesses larger than $30 \mathrm{~nm}$, much more relevant in practice, it appears that the hydrodynamic boundary conditions have no significant influence on the stability analysis, which a posteriori justifies the choice made in (14).

\section{Approximate model of the system}

In the present section, we will build an approximate model of our system by keeping only the most important (from the viewpoint of the instability threshold) physical phenomena and show that the corresponding results are in a very good agreement with the complete analysis presented above. Such a validated approximate form of the model has an advantage that it involves easy-touse analytical formulae. First, we will deduce an approximate reference solution. Then, we will show that the principal, solutal Marangoni mechanism of instability is well captured by the Pearson model with an appropriately defined Biot number, function of the wavenumber.

\subsection{Approximate form of the reference solution}

We have seen in section 3 that the Péclet numbers in the gas phase are quite small. It is easy to show that the temperature and concentration profiles for the reference solution are then linear in both the liquid and gas phases. In the liquid, these profiles are given by (29) and (31), whereas in the gas we now have $T_{g, r e f}=T_{b}+\left(T_{i, r e f}-T_{b}\right) \frac{H-z}{H-1}$ and $c_{g, r e f}=c_{i, r e f, g} \frac{H-z}{H-1}$ in lieu of (30) and (32), where it has been taken into account that $c_{t}=0$ and $T_{t}=T_{b}$. For simplicity, we also neglect the Soret effect here. Besides, the difference between the pressures $p_{i, g}$ and $p_{t}$ is neglected in Henry's law.

A simple estimation shows that the smallness of the Péclet numbers is intimately related to the smallness of the vapor concentration (interestingly enough, this can be observed already from

Table 3, where the two are very close indeed). We have $J^{d} \sim \rho_{g} D_{g} \frac{\partial c_{g}}{\partial z^{d}} \sim \rho_{g} D_{g} \frac{c_{i, g}}{d_{g}}$, while on the other hand $J^{d} \sim \rho_{g} w_{g}^{d}$. Consequently, $P e_{g, d i f f} \sim c_{i, g}$ indeed. Thus, consistent with using the linear profiles in the gas, we shall everywhere make simplifications corresponding to $c_{g} \ll 1$.

In particular, taking all this into account, the boundary conditions (19) and (23)-(25) become (for the reference profile)

$J_{\text {ref }}=-\frac{L e_{l}}{1-c_{l, \text { ref }}} \frac{\partial c_{l, \text { ref }}}{\partial z}=\frac{\rho D L e_{l}}{H-1} c_{g, r e f}=-\frac{\partial T_{l, \text { ref }}}{\partial z}-\frac{\lambda}{H-1}\left(T_{i, r e f}-T_{b}\right)$,

$c_{g, r e f}=\frac{c_{l, r e f}}{1+c_{l, r e f}\left(\delta_{M}-1\right)} \frac{K_{e}}{\delta_{M}^{\prime} p_{t}^{d}}$ 
all at $z=1$. This can be reduced to a closed-form boundary condition for $c_{l, r e f}$ :

$\frac{\partial c_{l, r e f}}{\partial z}=-\frac{\rho D}{H-1} \frac{c_{l, \text { ref }}\left(1-c_{l, \text { ref }}\right)}{1+c_{l, \text { ref }}\left(\delta_{M}-1\right)} \frac{K_{e}}{\delta_{M}^{\prime} p_{t}^{d}}$

at $z=1$. For the linear profile (31), this yields the following equation for $c_{i, r e f, l}$ :

$c_{i, r e f, l}-c_{b}=-\frac{\rho D}{H-1} \frac{c_{i, r e f, l}\left(1-c_{i, r e f, l}\right)}{1+c_{i, r e f, l}\left(\delta_{M}-1\right)} \frac{K_{e}}{\delta_{M}^{\prime} p_{t}^{d}}$,

which is in fact a quadratic equation, having the following (physical) solution

$c_{i, r e f, l}=\frac{c_{b}\left(1-\delta_{M}\right)+1+\widetilde{K}-\sqrt{\left(c_{b}\left(1-\delta_{M}\right)+1+\widetilde{K}\right)^{2}-4 c_{b}\left(1-\delta_{M}+\widetilde{K}\right)}}{2\left(1-\delta_{M}+\widetilde{K}\right)}$,

where

$\widetilde{K}=\frac{\rho D}{H-1} \frac{K_{e}}{\delta_{M}^{\prime} p_{t}^{d}}$.

Having calculated $c_{i, r e f, l}$ explicitely, some other quantities of interest are expressed as follows:

$c_{i, r e f, g}=\frac{c_{i, r e f, l}}{1+c_{i, r e f, l}\left(\delta_{M}-1\right)} \frac{K_{e}}{\delta_{M}^{\prime} p_{t}^{d}}$,

$J_{\text {ref }}=\frac{\rho D L e_{l}}{H-1} c_{i, r e f, g}$,

$T_{i, r e f}-T_{b}=-J_{\text {ref }}\left(1+\frac{\lambda}{H-1}\right)^{-1}$.

We have thus been able to deduce an approximate analytical expression of the reference solution under the assumption of small vapor concentration in the gas phase (and the Soret effect has also been neglected both in the liquid and in the gas).

Table 5 provides some numerical values of important physical quantities for the approximate solution. The comparison of this table with Table 3 shows a very good agreement with the full approach.

Table 5. Characteristics of the reference state (approximate analysis) for different values of $H$ $(10 \mathrm{wt} \%$ ethanol in water)

\begin{tabular}{lccccc}
\hline \hline \multicolumn{1}{c}{$H$} & $P e_{l, \text { diff }}$ & $P e_{g, \text { diff }}$ & $\left(T_{i, \text { ref }}^{d}-T_{b}^{d}\right)[K]$ & $\left(c_{i, \text { ref }, l}-c_{b}\right)$ & $\left(c_{i, \text { ref }, g}-c_{t}\right)$ \\
\hline 1.5 & $8.66 * 10^{-2}$ & $2.99 * 10^{-3}$ & $-1.65^{*} 10^{-1}$ & $-8.53 * 10^{-2}$ & $3.00^{*} 10^{-3}$ \\
2 & $7.63 * 10^{-2}$ & $5.27 * 10^{-3}$ & $-1.52^{*} 10^{-1}$ & $-7.43^{-1} 10^{-2}$ & $5.28^{*} 10^{-3}$ \\
4 & $5.15^{*} 10^{-2}$ & $1.07 * 10^{-2}$ & $-1.06^{*} 10^{-1}$ & $-4.88^{*} 10^{-2}$ & $1.07 * 10^{-2}$ \\
11 & $2.39 * 10^{-2}$ & $1.65 * 10^{-2}$ & $-4.97 * 10^{-2}$ & $-2.21^{*} 10^{-2}$ & $1.66^{*} 10^{-2}$ \\
101 & $3.02 * 10^{-3}$ & $2.09 * 10^{-2}$ & $-6.31 * 10^{-3}$ & $-2.73 * 10^{-3}$ & $2.09 * 10^{-2}$ \\
\hline \hline
\end{tabular}

An important particular case corresponds to large gas-to-liquid thickness ratios $(H \gg 1)$, which is quite of interest given that the critical liquid thicknesses (for the onset of instability) has turned out to be rather small (section 5). Besides, it is in this case that the limit of small variations $c_{l}$ across the liquid layer $\left(c_{b}-c_{i, r e f, l} \ll c_{b}\right)$ is eventually attained and that the full formulation used in this paper proves to be most self-consistent. For instance, this is in the sense that the material properties of the liquid have all been fixed at $c_{l}=c_{b}$ (section 2), whereas at least some of them are rather sensitive to $c_{l}$ : these include the diffusion coefficient [24], the Soret coefficient (11) 
and even $\gamma_{C}-$ cf. Table 2. Also, $P e_{l, \text { diff }}$ (neglected even in the full model by means of the quasistationary assumption) tends to zero as $H \rightarrow \infty$ together with $c_{b}-c_{i, r e f, l}$, whereas $P e_{g, \text { diff }}$ (included in the full model) tends to a constant together with $c_{i, r e f, g}$. For $H \gg 1$, the formulae (64)-(67) simplify just to

$c_{b}-c_{i, r e f, l}=\left(1-c_{b}\right) \frac{\rho D}{H-1} c_{i, r e f, g}$,

$c_{i, \text { ref }, g}=\frac{c_{b} / \delta_{M}^{\prime}}{1+c_{b}\left(\delta_{M}-1\right)} \frac{K_{e}}{p_{t}^{d}}$,

$J_{\text {ref }}=\frac{\rho}{1-\rho} w_{g, r e f}=\frac{\rho D L e_{l}}{H-1} c_{i, r e f, g}$,

$T_{i, r e f}-T_{b}=-\frac{\rho D L e_{l}}{H-1} c_{i, r e f, g}$,

which compare well (less than $3 \%$ difference) with the full approach at $H=101$. We find for $\left(T_{i, r e f}^{d}-T_{b}^{d}\right),\left(c_{i, r e f, l}-c_{b}\right)$ and $\left(c_{i, r e f, g}-c_{t}\right)$ the values $-6.50 * 10^{-3} \mathrm{~K},-2.80^{*} 10^{-3}$ and $2.15^{*} 10^{-2}$ from (63)-(71), as compared to $-6.30 * 10^{-3} \mathrm{~K},-2.74 * 10^{-3}$ and $2.07 * 10^{-2}$ for the full model.

\subsection{Stability analysis: Pearson-like model}

The stability analysis based on the full model has shown that the solutal Marangoni effect is by far the most important instability mechanism. In the present section, we will thus neglect all the other effects when analyzing the stability. We will also continue assuming that convection in the gas can be neglected (neglecting $w_{g, r e f}$ and $W_{g}$ in equation (46)). The term with $\sigma$ in (46) can be neglected on the basis of $D \gg 1$ (assuming that the complex growth rate of the perturbation is associated with the diffusion time scale in the liquid). Thus, equation (46) becomes

$D_{z}^{2} C_{g}-k^{2} C_{g}=0$.

Keeping in mind that $C_{g}=0$ at the top boundary, the solution is

$C_{g}=C_{i, g} \frac{\sinh (k(z-H))}{\sinh (k(1-H))}$.

The following boundary conditions can be deduced from (23)-(25) within the simplifications discussed in the previous subsection:

$\frac{1}{1-c_{l}} \frac{\partial c_{l}}{\partial z}=\rho D \frac{\partial c_{g}}{\partial z}, \quad c_{g}=\frac{c_{l}}{1+c_{l}\left(\delta_{M}-1\right)} \frac{K_{e}}{\delta_{M}^{\prime} p_{t}^{d}}$

at $z=1$, which have already been used when looking for the approximate solution for the reference profile. Now for the perturbations, they become

$\frac{1}{1-c_{l, \text { ref }}} \frac{\partial C_{l}}{\partial z}+\frac{C_{l}}{\left(1-c_{l, r e f}\right)^{2}} \frac{\partial c_{l, r e f}}{\partial z}=\rho D \frac{\partial C_{g}}{\partial z}, \quad C_{g}=\frac{C_{l}}{\left[1+c_{l, r e f}\left(\delta_{M}-1\right)\right]^{2}} \frac{K_{e}}{\delta_{M}^{\prime} p_{t}^{d}}$

at $z=1$. Using (63) and (72), this can be reduced to the form

$\partial C_{l} / \partial z+B i_{s} C_{l}=0$,

with a solutal "Biot number" given by

$B i_{s}(k, H)=\rho D \frac{K_{e}}{\delta_{M}^{\prime} p_{t}^{d}}\left\{\frac{1-c_{i, r e f, l}}{\left[1+c_{i, r e f, l}\left(\delta_{M}-1\right)\right]^{2}} k \operatorname{coth}(k(H-1))-\frac{c_{i, r e f, l}}{(H-1)\left[1+c_{i, r e f, l}\left(\delta_{M}-1\right)\right]}\right\}$.

With (73), our formulation for the perturbation of the concentration field in the liquid layer is represented in Pearson's terms [3]. Invoking besides the smallness of the evaporation Péclet 
number in the liquid and neglecting the gas viscosity, we recover an equivalent form of Pearson's formulation [3]. Thus, we can simply recur to his result for the marginal curve in order to describe our solutal Marangoni instability:

$M s^{*}(k, H)=8 k \frac{\left[k \cosh (k)+B i_{S}(k, H) \sinh (k)\right][\sinh (k) \cosh (k)-k]}{\sinh ^{3}(k)-k^{3} \cosh (k)}$.

Here note that $M s^{*}$ is the "true" solutal Marangoni number as is defined in (61). Fig. 11 represents (75) with (74) for a number of $H$ values, where $c_{i, r e f, l}$ is taken according to (64).

(a)

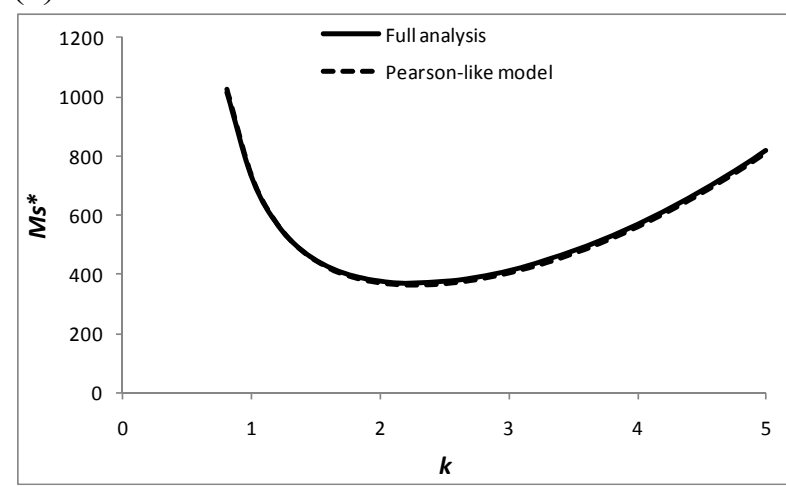

(c)

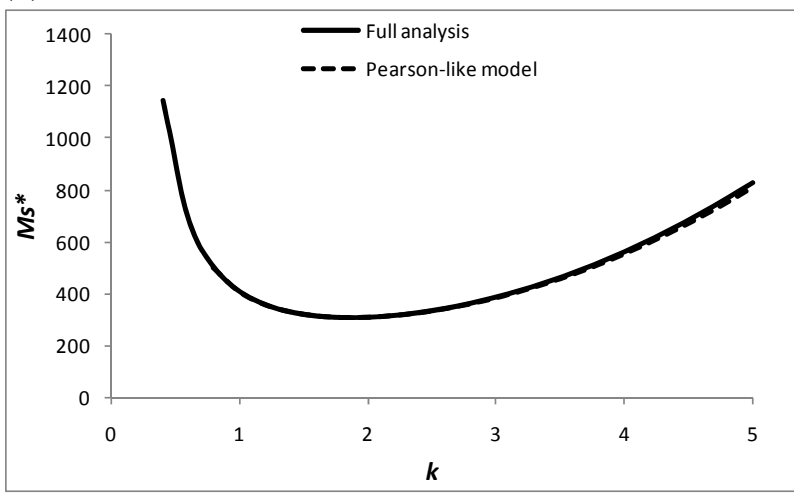

(b)

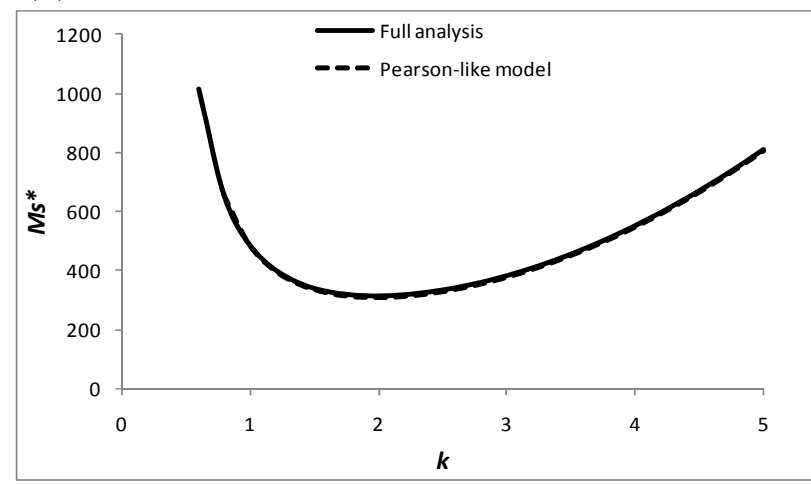

(d)

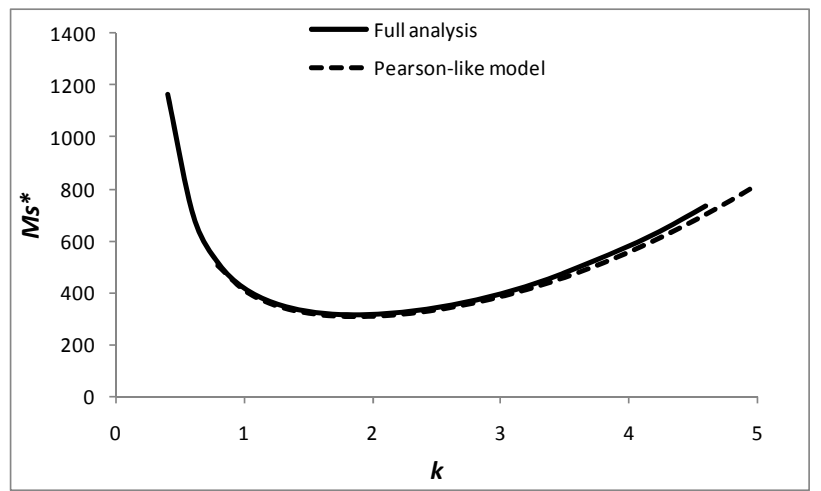

Fig. 11. The marginal curves in terms of the "true" solutal Marangoni number versus the wave number: comparison between the complete model of the present paper and the Pearson-like simplified model for $\mathrm{H}=1.5$ (a), $\mathrm{H}=2$ (b), $\mathrm{H}=11$ (c) $\mathrm{H}=101$ (d) (10 wt\% ethanol in water)

It shows an excellent agreement of the Pearson-like model with the full analysis. In particular, this suggests that convection in the gas phase is of minor importance indeed for different values of $H$. On the other hand, this underscores the importance of taking the Biot number as a function of the wavenumber of the perturbation as in (74) and not just the one defined by the uniform (reference) state, formally corresponding to $k \rightarrow 0$ in (74), as it is sometimes the case in the literature [18]. Fig. 12(a) shows the critical liquid thickness as a function of the gas thickness for both models, while Fig. 12(b) presents the critical Marangoni number as a function of the gas thickness for both models as well, which also manifests an excellent agreement. 
(a)

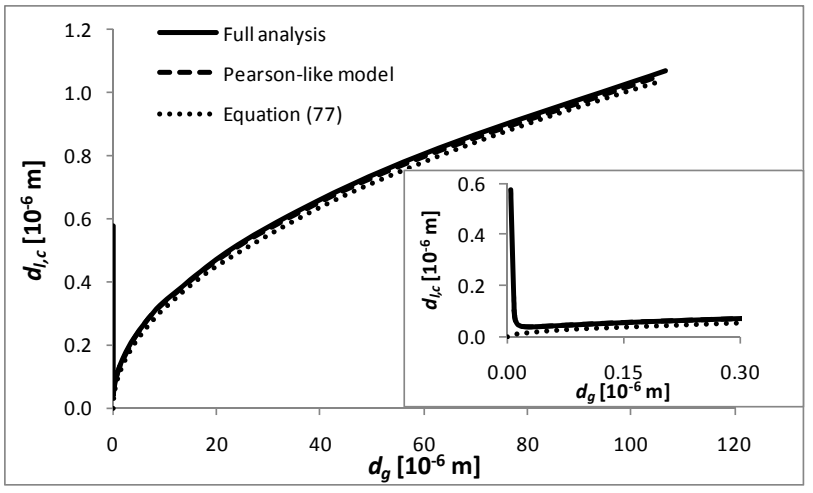

(b)

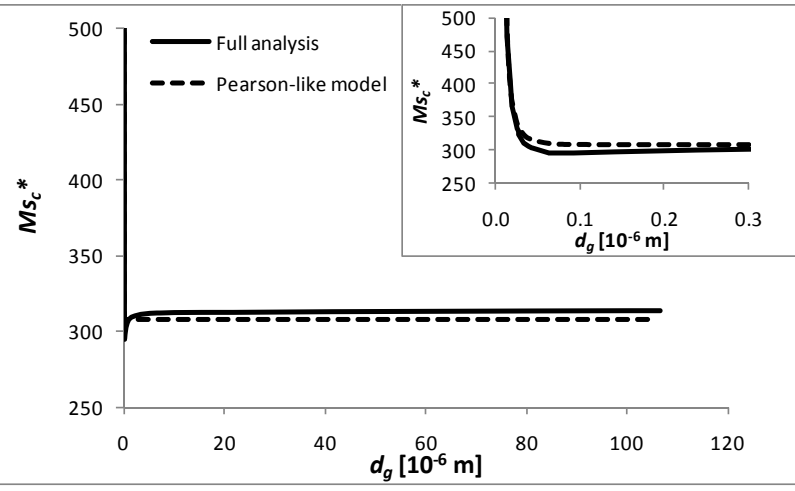

Fig. 12. The critical liquid thickness (a) and the critical "true" solutal Marangoni number (b) versus the gas layer thickness: comparison with the Pearson-like model $(10 \mathrm{wt} \%$ ethanol in water)

The Pearson-like model can also help understanding the tendencies observed for the critical liquid layer thickness as $d_{g}$ (and $H$ ) are increased, cf. Fig. 5(a,b) and Fig. 12(a). It can be observed from these figures, that at large $d_{g}$ (and $H$ ), a certain constant is attained for $M s_{C}^{*}$. This phenomenon can now be explained. In an important special case $H \gg 1$ (cf. the end of subsection 6.1), the Biot number (74) adopts a particularly simple form:

$B i_{s}(k)=\rho D\left(1-c_{b}\right) \frac{1}{\left[1+c_{b}\left(\delta_{M}-1\right)\right]^{2}} \frac{K_{e}}{\delta_{M}^{\prime} p_{t}^{d}} k$.

Accordingly, the critical value $M s_{c}^{*}$ (the minimum of the neutral curve (75) with (76)) ceases to depend on the thicknesses, which is what one can observe in Fig. 12(b). Actually, this is the case not only for the minimum, but also for the neutral curve as a whole, except for small $k$ values, in line with what has already been pointed out when discussing Fig. 8. As $M s^{*} \equiv M s\left(c_{b}-c_{i, r e f, l}\right)$, whereas $\left(c_{b}-c_{i, r e f, l}\right)$ is given by (68) with (69) in the limit $H \rightarrow \infty$, one can now see that $d_{l, c} \sim H$ or, which is the same, $d_{l, c} \sim \sqrt{d_{g}}$ as $H \rightarrow \infty \quad\left(d_{g} \rightarrow \infty\right)$ in accordance with the observed tendencies in Figs. 5(a), 5(b) and 12(a). More specifically, the Pearson-like model yields $M s_{c}^{*}=$ 307.5 as $H \rightarrow \infty$, so one obtains $\left(c_{b}=0.1\right)$

$\frac{d_{l, c}^{2}}{d_{g}}=307.5 \frac{D_{l}^{2} \mu_{l} \rho_{l} \delta_{M}^{\prime}}{\gamma_{C} D_{g} \rho_{g}} \frac{1+c_{b}\left(\delta_{M}-1\right)}{c_{b}\left(1-c_{b}\right)} \frac{p_{t}^{d}}{K_{e}} \approx 1.02 \times 10^{-8} \mathrm{~m} \quad$ as $\quad H \rightarrow \infty$,

which is shown in Fig. 12(a) by a dotted line.

Another phenomenon observed in Fig. 5, and also present in Fig. 12(a), is the minimum in $d_{l, c}$ for small $d_{g}$ (and $H$ ). This can be explained as follows. For large $d_{g}$, the evaporation flux decreases since the pathway of ethanol across the gas phase becomes longer. Therefore, the ethanol mass fraction difference between the bottom of the liquid and the interface decreases, with $c_{i, r e f, l} \rightarrow c_{b}$ as $d_{g} \rightarrow \infty(H \rightarrow \infty)$. Accordingly, an instability can appear in the system only if the thickness of the liquid layer is increased. Note that this critical liquid thickness even tends to grow with $d_{g}$ boundlessly since the critical Marangoni number tends to a constant for large $d_{g}$, as discussed above. On the other hand, as $d_{g}$ is decreased, the ethanol mass fraction 
difference between the bottom of the liquid and the interface increases but reaches a saturation. The mass fraction of ethanol at the interface becomes almost constant (zero if $c_{t}=0$ ) and the Marangoni effect tends to disappear, which also results in $d_{l, c} \rightarrow \infty$ as $d_{g}$ is decreased. The minimum in $d_{l, c}$ in Fig. 5 or Fig. 12a is thus a consequence of an increasing $d_{l, c}$ for both large and small $d_{g}$. As an additional comment, it is noteworthy to mention that a minimum also appears in Fig. 12(b), the physical interpretation of which is more delicate. This minimum comes from the dependence of the Biot number (74) on $H$. More precisely, this Biot number has itself a minimum with respect to $H$ due to evaporation and the resulting dependence of $c_{i, r e f, l}$ with respect to $H$.

To finish this section, let us briefly comment on the flat interface assumption considered in our model. Surface deformation can be neglected if the capillary number is small $(\mathrm{Ca} \ll 1)$ and if the Galileo number is large $(G a \gg 1)$ [6]. The first condition, irrespective of the second one (albeit the latter can also help if satisfied), ensures that the surface deformation is negligible for the finite- $k$ modes we have studied here, whereas the second one guarantees the absence of low- $k$ surface deformation modes of instability. Defining the "solutal" capillary and Galileo numbers in the liquid as $C s=\mu_{l} D_{l} / \sigma_{l} d_{l}$ and $G s=g d_{l}^{3} / \nu_{l} D_{l}$, it is easy to show that for our typical critical thicknesses the first condition is satisfied, but not the second. Hence, our analysis of the finite- $k$ modes turns out to be consistent, even though the surface deformation modes, characterized by low wavenumbers, can actually become unstable first. The nonlinear competition between these surface modes and the finite wave-length instability has already been studied in the literature [6, 29]. Let us only mention that since the growth rate of the finite wave-length mode is much larger than that of the surface mode, the patterned instability can occur even on the background of a large scale surface deformation, appearing on a larger time scale. It is from this viewpoint that the analysis of the present paper restricted to the finite wave-length modes is justified. Another viewpoint is that the assessments of various mechanisms of instability made here can be useful as estimations in situations when the surface modes of instability do not occur at all.

\section{Approximate model with water evaporation}

So far, water evaporation has been neglected in the present paper. Here, we incorporate it into the stability analysis, albeit just within the approximate approach as developed in the previous section. Before embarking on the stability analysis, we describe the modifications in the mathematical formulation of the full problem brought about by taking water evaporation into account.

\subsection{Changes to the full model on account of water volatility}

The equations for the liquid phase do not change. The modifications are rather related to the gas phase, which is now described by an additional dependent variable - the mass fraction of water vapor. Thus, e.g., equation (2) becomes

$\rho_{g}=\rho_{g, 0}\left\{1-\alpha_{g}\left(T_{g}^{d}-T_{g, 0}^{d}\right)-\varepsilon_{g 2}\left(c_{g 2}-c_{g 2,0}\right)-\varepsilon_{g 1}\left(c_{g 1}-c_{g 1,0}\right)\right\}$. 
The subscripts " 1 " and " 2 " refer in this subsection to the solvent (water) and solute (ethanol), respectively. These new notations are needed only in the three-component gas phase and we will keep the previous ones in the liquid. The counterparts of (8) and (10) are now written as $\frac{\partial \vec{v}_{g}}{\partial t}=-\left(\vec{v}_{g} \cdot \nabla\right) \vec{v}_{g}+\operatorname{Pr}_{l}\left\{-\rho^{-1} \nabla p_{g}-G a_{l} 1_{z}+v \nabla^{2} \vec{v}_{g}+\alpha R a_{l} T_{\mathrm{g}} 1_{z}+\varepsilon R s_{l} L e_{l}\left(c_{g 2}-c_{g 2,0}\right) 1_{z}+\right.$ $\left.\delta_{\varepsilon} \varepsilon R s_{l} L e_{l}\left(c_{g 1}-c_{g 1,0}\right) 1_{z}\right\}$,

$\frac{\partial c_{g 1}}{\partial t}=-\left(\vec{v}_{g} \cdot \nabla\right) c_{g 1}+\delta_{D} D L e_{l} \nabla^{2} c_{g 1}$,

$\frac{\partial c_{g 2}}{\partial t}=-\left(\vec{v}_{g} \cdot \nabla\right) c_{g 2}+D L e_{l} \nabla^{2} c_{g 2}$,

with the new species balance equation (80) added for the water vapor. The following dimensionless numbers are to be (re)defined:

$\varepsilon=\frac{\varepsilon_{g 2}}{\varepsilon_{l}}, D=\frac{D_{g 2}}{D_{l}}, \delta_{\varepsilon}=\frac{\varepsilon_{g 1}}{\varepsilon_{g 2}}, \delta_{D}=\frac{D_{g 1}}{D_{g 2}}$.

The gas-phase mass fluxes in (12) become

$\vec{J}_{g 1}=-\rho D L e_{l} \delta_{D} \nabla c_{g 1}, \quad \vec{J}_{g 2}=-\rho D L e_{l} \nabla c_{g 2}$,

As for the boundary conditions, no changes occur at $z=0$, while at the top boundary $z=H$ we now have

$c_{g 1}=c_{t 1}, \quad c_{g 2}=c_{t 2}$,

where $c_{t 1}$ and $c_{t 2}$ are the known "ambient" values. At $z=h(t)$, the counterpart of (19) is

$-\frac{\partial T_{l}}{\partial z}+\lambda \frac{\partial T_{g}}{\partial z}=J_{2}+\delta_{L} J_{1}$

where

$\delta_{L}=\frac{L_{2}}{L_{1}}$,

whereas $J_{1}$ and $J_{2}$ are the water and ethanol evaporation fluxes respectively, with the total evaporation flux now being

$J=J_{1}+J_{2}$.

Of course, $J$ is still the one that appears in (15) and (17). Expressing the continuity of individual fluxes $J_{1}$ and $J_{2}$ of the solvent and of the solute on each side of the interface, as well as the vanishing of the inert gas flux (no absorption), the following relations are obtained at $z=h(t)$ :

$J_{1}=J\left(1-c_{l}\right)+L e_{l}\left(\frac{\partial c_{l}}{\partial z}+\psi_{S} \frac{\partial T_{l}}{\partial z}\right)=J c_{g 1}-\rho D \delta_{D} L e_{l} \frac{\partial c_{g 1}}{\partial z}$,

$J_{2}=J c_{l}-L e_{l}\left(\frac{\partial c_{l}}{\partial z}+\psi_{S} \frac{\partial T_{l}}{\partial z}\right)=J c_{g 2}-\rho D L e_{l} \frac{\partial c_{g 2}}{\partial z}$,

$J\left(1-c_{g 1}-c_{g 2}\right)=-\rho D L e_{l}\left(\delta_{D} \frac{\partial c_{g 1}}{\partial z}+\frac{\partial c_{g 2}}{\partial z}\right)$

Of these six relations (Eq. (85), together with the above five), only four are independent. These can be rendered in the form

$J=-\frac{L e_{l}}{1-c_{l}-c_{g 1}}\left\{\left[\frac{\partial c_{l}}{\partial z}+\psi_{S} \frac{\partial T_{l}}{\partial z}\right]+\rho \delta_{D} D \frac{\partial c_{g 1}}{\partial z}\right\}$,

$J=-\frac{\rho D L e_{l}}{1-c_{g 1}-c_{g 2}}\left\{\frac{\partial c_{g 2}}{\partial z}+\delta_{D} \frac{\partial c_{g 1}}{\partial z}\right\}$,

$J_{1}=J c_{g 1}-\rho D \delta_{D} L e_{l} \frac{\partial c_{g 1}}{\partial z}$,

$J_{2}=J c_{g 2}-\rho D L e_{l} \frac{\partial c_{g 2}}{\partial z}$, 
all at $z=h(t)$, where (86) and (87) can be recognized as the counterparts of (23) and (24), respectively. With (86) and (87), the number of interface boundary conditions is thus far exactly equal to that in the case of no water evaporation (subsection 2.2). However, two supplementary conditions, (88) and (89), are attributed to the newly introduced quantities $J_{1}$ and $J_{2}$.

The counterpart of (25), Henry's law, is

$\frac{c_{g 2} \delta_{M}^{\prime \prime}}{1+c_{g 1}\left(\delta_{M}^{\prime}-1\right)+c_{g 2}\left(\delta_{M}^{\prime \prime}-1\right)}=\frac{c_{l} \delta_{M}}{1+c_{l}\left(\delta_{M}-1\right)} \frac{K_{e}^{n}}{p_{g}}$.

at $z=h(t)$.

As now it is not only the solute but also the solvent that evaporates, an additional interface condition is needed, which is given by Raoult's law (dilute assumption, consistent with using Henry's law for the solute) [22]:

$y_{g 1} p_{g}^{d}=\left(1-y_{l}\right) p_{\text {sat } 1}$,

where $p_{\text {sat } 1}$ is the saturation pressure of a pure solvent, a function of the interface temperature (just as for the Henry coefficient, this temperature dependence is neglected in the present paper in view of the very small temperature variations). In terms of mass fractions, Raoult's law can be rewritten as

$\frac{c_{g 1} \delta_{M}^{\prime}}{1+c_{g 1}\left(\delta_{M}^{\prime}-1\right)+c_{g 2}\left(\delta_{M}^{\prime \prime}-1\right)}=\frac{\left(1-c_{l}\right)}{1+c_{l}\left(\delta_{M}-1\right)} \frac{p_{s a t 1}^{n}}{p_{g}}$

at $z=h(t)$, where $p_{s a t 1}^{n}$ is the non-dimensional saturation pressure (again, using $\mu_{l} \kappa_{l} / d_{l}^{2}$ as scale).

To sum up, the formulation of section 2 is modified as follows: (2) is replaced by (78), (8) by (79), (10) by (80) and (81), the third expression (12) by (82), the second condition (14) by (83), (19) by (84), (23) by (86), (24) by (87), (25) by (90), and besides we have to consider (88), (89) and (91).

\subsection{When is solvent (water) evaporation negligible?}

The non-volatile solvent case is formally recovered when $p_{s a t 1}=c_{t 1}=0$ (then, $c_{g 1}=J_{1}=0$ ). For the water-ethanol system at $300 \mathrm{~K}, p_{\text {sat } 1}$ is one order of magnitude smaller than $K_{e}$ (see Table 1 and Table 6), which plays in favor of the main approach (neglecting solvent evaporation) used here before the present section. Yet, as one can appreciate from (90) and (91), for $c_{l} \sim 0.1$ (10 $\mathrm{wt} \%$ ethanol in water), the gas concentrations of ethanol and water are comparable to one another. To what extent this alters the results obtained while neglecting water evaporation is one of the questions raised in the present section. Table 6 presents the physical quantities that are needed in addition to Tables 1 and 2 when considering water evaporation. The explanations and sources of these values can be found in Appendix A.

Table 6. Supplementary physical properties needed in the case of water evaporation

\begin{tabular}{cl}
\hline Physical property & Value \\
\hline$D_{g 1}$ & $2.54 * 10^{-5} \mathrm{~m}^{2} / \mathrm{s}$ \\
$p_{\text {sat } 1}$ & $3.567 * 10^{3} \mathrm{~Pa}$
\end{tabular}




$\begin{array}{ll}\varepsilon_{g 1} & 6.11 * 10^{-1} \\ L_{1} & 2.439 * 10^{6} \mathrm{~J} / \mathrm{kg}\end{array}$

On the other hand, it has been suggested section 2 that the effects of water evaporation could be mitigated by choosing the "right" ambient humidity. To begin with, note that the evaporation ceases at all if the ambient vapor concentrations are $c_{t 1}=c_{t 1 *}$ and $c_{t 2}=c_{t 2 *}$ such that

$\frac{c_{t 2 *} \delta_{M}^{\prime \prime}}{1+c_{t 1 *}\left(\delta_{M}^{\prime}-1\right)+c_{t 2 *}\left(\delta_{M}^{\prime \prime}-1\right)}=\frac{c_{b} \delta_{M}}{1+c_{b}\left(\delta_{M}-1\right)} \frac{K_{e}}{p_{t}^{d}}, \quad \frac{c_{t 1 x^{\prime}} \delta_{M}^{\prime}}{1+c_{t 1 *}\left(\delta_{M}^{\prime}-1\right)+c_{t 2 *}\left(\delta_{M}^{\prime \prime}-1\right)}=\frac{\left(1-c_{b}\right)}{1+c_{b}\left(\delta_{M}-1\right)} \frac{p_{s a t 1}}{p_{t}^{d}}$

as given by Henry's and Raoult's laws (90) and (91) at $c_{l}=c_{b}\left(c_{t 1 *}\right.$ and $c_{t 2 *}$ are actually the saturation vapor concentrations for the solution with $c_{l}=c_{b}$ ). The case $c_{t 2}=c_{t 2 *}$ is of no particular interest here, however, and the parameter $c_{t 2}$ will be regarded as varying in a wide range (for the most part, we shall keep considering that the ambient air is free of ethanol vapor, $c_{t 2}=0$ ). But with $c_{t 2} \neq c_{t 2 *}$, water evaporation does not cease completely even for $c_{t 1}=c_{t 1 *}$. Yet, its effect is expected to be reduced to a minimum. So $c_{t 1}=c_{t 1 *}$ as given by equations (92) will be used as a test case for the mentioned "right" humidity, irrespective of the value of $c_{t 2}$.

\subsection{Approximate approach}

The case with water evaporation is not considered in the present paper other than in the framework of the approximate approach introduced in section 6. The approximation is based upon neglecting the convective effects in the gas phase. It is justified, on the one hand, by the assumed smallness of vapor concentrations (here $c_{g 1}$ and $c_{g 2}$ ) which also implies the smallness of the (evaporation) Peclet number in the gas (cf. subsection 6.1), and on the other hand, by the diffusivities in the gas being several orders of magnitude larger than those in the liquid (cf. subsection 6.2). The difference between the pressure $p_{i, g}$ at the interface and the top pressure $p_{t}$ is also neglected in Henry's and Raoult's laws, for the overall consistency of the analysis..

Note, however, that the approximate approach used here disregards a possible instability mechanism associated with the gas layer. Indeed, as water vapor (unlike the ethanol vapor) is lighter than the air (note a positive value of $\varepsilon_{g 1}$ in Table 6), a buoyancy-driven instability can be expected for sufficiently large gas-layer thicknesses. For instance, to have the gas solutal Rayleigh number $\varepsilon_{g 1} g d_{g}^{3} / v_{g} D_{g}$ of the order of 100 , one must have $d_{g} \sim 0.7 \mathrm{~cm}$. In reality, this destabilizing effect due to water evaporation is countered by the similar stabilizing effects due to ethanol evaporation (the ethanol vapor is heavier than the air) and the thermal gradient (here directed upwards, hence opposing to convection in the gas), therefore leading to an even larger critical value of $d_{g}$. Thus, omitting consideration of this possible instability mechanism appears justified for the small gas thicknesses considered here: the critical liquid layer thicknesses are rather small and, unless we consider very large values of $H(H \gg 101)$, the gas layer thicknesses also remain limited. Yet this point would have to be reconsidered, should the analysis be applied to larger thicknesses.

Thus, we have the equations

$\nabla^{2} T_{g}=0, \quad \nabla^{2} c_{g 1}=0, \quad \nabla^{2} c_{g 2}=0$ 
in the bulk of the gas phase, whereas (86)-(91) get simplified to

$J=-\frac{L e_{l}}{1-c_{l}}\left\{\left[\frac{\partial c_{l}}{\partial z}+\psi_{S} \frac{\partial T_{l}}{\partial z}\right]+\rho D \delta_{D} \frac{\partial c_{g 1}}{\partial z}\right\}=-\rho D L e_{l}\left\{\frac{\partial c_{g 2}}{\partial z}+\delta_{D} \frac{\partial c_{g 1}}{\partial z}\right\}$,

$J_{2}=-\rho D L e_{l} \frac{\partial c_{g 2}}{\partial z}, \quad J_{1}=-\rho D \delta_{D} L e_{l} \frac{\partial c_{g 1}}{\partial z}$,

$c_{g 2}=\frac{c_{l}}{1+c_{l}\left(\delta_{M}-1\right)} \frac{K_{e}}{\delta_{M}^{\prime} p_{t}^{d}}, \quad c_{g 1}=\frac{1-c_{l}}{1+c_{l}\left(\delta_{M}-1\right)} \frac{p_{s a t 1}}{\delta_{M}^{\prime} p_{t}^{d}}$

all at $z=h(t)$. Besides, $c_{t 1 *}$ is now given by (cf. (92))

$c_{t 1 *}=\frac{1-c_{b}}{1+c_{b}\left(\delta_{M}-1\right)} \frac{p_{s a t 1}}{\delta_{M}^{\prime} p_{t}^{d}}$.

Note also that the second equality in (93) can be rewritten in the following useful form:

$\frac{\partial c_{l}}{\partial z}+\psi_{S} \frac{\partial T_{l}}{\partial z}=\rho D\left(1-c_{l}\right) \frac{\partial c_{g 2}}{\partial z}-\rho D \delta_{D} c_{l} \frac{\partial c_{g 1}}{\partial z}$

at $z=h(t)$.

\subsection{Reference profiles (approximate approach)}

We proceed similarly to subsection 6.1 using the formulae of subsection 7.3. Within the approximation in question, the reference profiles in the gas phase are just linear: $T_{g, r e f}=T_{b}+$ $\left(T_{i, r e f}-T_{b}\right) \frac{H-Z}{H-1}, c_{g 2, r e f}=c_{t 2}+\left(c_{i, r e f, g 2}-c_{t 2}\right) \frac{H-Z}{H-1}$ and $c_{g 1, r e f}=c_{t 1}+\left(c_{i, r e f, g 1}-c_{t 1}\right) \frac{H-Z}{H-1}$, where it has been taken into account that $T_{t}=T_{b}$. With these linear profiles, using (84), (94), (95) and (97), one can derive the following closed-form interface boundary conditions for the liquid layer:

$L e_{l}\left(\frac{\partial c_{l, \text { ref }}}{\partial z}+\psi_{S} \frac{\partial T_{l, \text { ref }}}{\partial z}\right)=-\left(1-c_{l, \text { ref }}\right) J_{2, \text { ref }}+c_{l, \text { ref }} J_{1, \text { ref }}$,

$\frac{\partial T_{l, \text { ref }}}{\partial z}+\frac{\lambda}{H-1}\left(T_{l, \text { ref }}-T_{b}\right)+J_{2, \text { ref }}+\delta_{L} J_{1, \text { ref }}=0$,

$J_{1, \text { ref }}=\frac{\rho D \delta_{D} L e_{l}}{H-1}\left(\frac{1-c_{l, r e f}}{1+c_{l, \text { ref }}\left(\delta_{M}-1\right)} \frac{p_{\text {sat1 } 1}}{\delta_{M}^{d} p_{t}^{d}}-c_{t 1}\right)$,

$J_{2, \text { ref }}=\frac{\rho D L e_{l}}{H-1}\left(\frac{c_{l, r e f}}{1+c_{l, \text { ref }}\left(\delta_{M}-1\right)} \frac{K_{e}}{\delta_{M}^{\prime} p_{t}^{d}}-c_{t 2}\right)$.

at $z=h(t)$. These, together with (cf. (15) and (85))

$J_{\text {ref }}=J_{1, \text { ref }}+J_{2, \text { ref }}$,

$\frac{d h}{d t}=-J_{\text {ref }}+w_{l, \text { ref }}$

represent a full set of interfacial boundary conditions for the resolution of the reference-profile problem in the liquid layer within effectively a one-layer formulation.

For the particular configuration studied in the present paper and within the quasi-stationary approach (see section 3), the reference profiles in the liquid are of the form (26), (29) and (31), where one can formally and without loss of generality set $h=1$ whereas $\dot{h}=-J_{\text {ref }}$. Using these profiles in (98)-(101), one obtains a system of four algebraic equations for the four unknown quantities $c_{i, r e f, l}, T_{i, r e f, l}, J_{1, \text { ref }}$ and $J_{2, \text { ref }}$. Subsequently, $J_{\text {ref }}$ and $\dot{h}$ can be determined from (102) and from $\dot{h}=-J_{r e f}$, respectively. In principle, the saturation pressure and the Henry coefficient in (100) and (101) are (known) functions of the interface temperature $T_{i, r e f, l}$. However, as already said, this dependence is neglected in the present paper and we consider 
them just as constants. Now, if similarly to section 6 we neglect also the Soret effect, the problem for the concentration field gets decoupled from that of the temperature, and $c_{i, r e f, l}$ satisfies just a quadratic equation (obtained from (98) with (31), (100), (101) and $\psi_{S} \equiv 0$ ):

$c_{i, r e f, l}-c_{b}=-\frac{\rho D}{H-1} \frac{c_{i, r e f, l}\left(1-c_{i, r e f, l}\right)}{1+c_{i, r e f, l}\left(\delta_{M}-1\right)} \frac{K_{e}-\delta_{D} p_{\text {sat } 1}}{\delta_{M}^{\prime} p_{t}^{d}}+\frac{\rho D}{H-1}\left(1-c_{i, r e f, l}\right) c_{t 2}-\frac{\rho D \delta_{D}}{H-1} c_{i, r e f, l} c_{t 1}$,

The solution, i.e. the counterpart of (64), is

$c_{i, r e f, l}=\frac{A_{1}-\sqrt{A_{1}^{2}-4 A_{0} A_{2}}}{2 A_{2}}$

with

$A_{2}=\widetilde{K}+\left(1-\delta_{M}\right)\left(1+Q_{1}+Q_{2}\right)$,

$A_{1}=\widetilde{K}+c_{b}\left(1-\delta_{M}\right)+1+Q_{2}\left(2-\delta_{M}\right)+Q_{1}$,

$A_{0}=c_{b}+Q_{2}$,

$Q_{1}=\frac{\rho D \delta_{D}}{H-1} c_{t 1}$,

$Q_{2}=\frac{\rho D}{H-1} c_{t 2}$,

$\widetilde{K}=\frac{\rho D}{H-1} \frac{K_{e}-\delta_{D} p_{s a t 1}}{\delta_{M}^{\prime} p_{t}^{d}}$

With $c_{i, r e f, l}$ obtained, the other quantities of interest are calculated in a straightforward way. For instance, in accordance with (100) and (101), we have

$J_{1, \text { ref }}=\frac{\rho D \delta_{D} L e_{l}}{H-1}\left(\frac{1-c_{i, r e f, l}}{1+c_{i, r e f, l}\left(\delta_{M}-1\right)} \frac{p_{s a t 1}}{\delta_{M}^{\prime} p_{t}^{d}}-c_{t 1}\right)$,

$J_{2, r e f}=\frac{\rho D L e_{l}}{H-1}\left(\frac{c_{i, r e f, l}}{1+c_{i, r e f, l}\left(\delta_{M}-1\right)} \frac{K_{e}}{\delta_{M}^{\prime} p_{t}^{d}}-c_{t 2}\right)$,

which can be subsequently used in (102) to obtain $J_{r e f}$. The latter permits in turn calculating $w_{g, \text { ref }}$ and $\dot{h}$, by means of (27) and of $\dot{h}=-J_{\text {ref }}$. Accordingly, $P e_{l, \text { diff }}$ and $P e_{g, \text { diff }}$ can be calculated as is done in section 3. The interface temperature is computed by using (29) in (99):

$T_{i, r e f}-T_{b}=-\left(J_{2, r e f}+\delta_{L} J_{1, r e f}\right)\left(1+\frac{\lambda}{H-1}\right)^{-1}$.

At last, the interface concentrations on the gas side are expressed with the help of (95):

$c_{i, r e f, g 2}=\frac{c_{i, r e f, l}}{1+c_{i, r e f, l}\left(\delta_{M}-1\right)} \frac{K_{e}}{\delta_{M}^{\prime} p_{t}^{d}}, \quad c_{i, r e f, g 1}=\frac{1-c_{i, r e f, l}}{1+c_{i, r e f, l}\left(\delta_{M}-1\right)} \frac{p_{s a t 1}}{\delta_{M}^{\prime} p_{t}^{d}}$.

The reference state characteristics calculated in this way are provided in Table 7 for a number of $H$ values in the case $c_{t 1}=0$ (dry ambient air) and $c_{t 2}=0$. In order to show that there exists a case where water volatility plays only a minor role, the same characteristics are also shown for $c_{t 1}=c_{t 1 *}$ (the "right" ambient humidity, equation (96)) and $c_{t 2}=0$ in Table 8 . Tables 7 and 8 are the counterparts of Tables 3 and 5 (full approach and approximate analysis, respectively, without water evaporation).

Table 7. Characteristics of the reference state (approximate analysis with water evaporation) for different values of $H$ with $c_{t 1}=0$ and $c_{t 2}=0$ (10 $\mathrm{wt} \%$ ethanol in water)

\begin{tabular}{lllllll}
\hline$H$ & $P e_{l, \text { diff }}$ & $P e_{g, \text { diff }}$ & $\left(T_{i, r e f}^{d}-T_{b}^{d}\right)$ & $\left(c_{i, r e f, l}-c_{b}\right)$ & $\left(c_{i, r e f, g 1}-c_{t 1}\right)$ & $\left(c_{i, r e f, g 2}-c_{t 2}\right)$ \\
\hline
\end{tabular}




\begin{tabular}{lllllll}
\hline \hline \multicolumn{5}{c}{$[K]$} \\
\hline 1.5 & $1.44 * 10^{0}$ & $4.96^{*} 10^{-2}$ & $-5.65 * 10^{0}$ & $-8.18^{*} * 10^{-2}$ & $2.17^{*} 10^{-2}$ & $3.73 * 10^{-3}$ \\
2 & $7.53 * 10^{-1}$ & $5.20 * 10^{-2}$ & $-3.02 * 10^{0}$ & $-6.90 * 10^{-2}$ & $2.16^{*} 10^{-2}$ & $6.39 * 10^{-3}$ \\
4 & $2.76^{*} 10^{-1}$ & $5.72 * 10^{-2}$ & $-1.08 * 10^{0}$ & $-4.23 * 10^{-2}$ & $2.13 * 10^{-2}$ & $1.21 * 10^{-2}$ \\
11 & $8.99 * 10^{-2}$ & $6.21 * 10^{-2}$ & $-3.42 * 10^{-1}$ & $-1.79 * 10^{-2}$ & $2.11 * 10^{-2}$ & $1.75^{*} 10^{-2}$ \\
101 & $9.46^{*} 10^{-3}$ & $6.53 * 10^{-2}$ & $-3.53 * 10^{-2}$ & $-2.12 * 10^{-3}$ & $2.10^{*} 10^{-2}$ & $2.11 * 10^{-2}$ \\
\hline \hline
\end{tabular}

Comparing Table 7 with Table 3, one can observe that allowing water to evaporate results in an appreciable increase of the Péclet numbers and of the temperature drop at the interface. This is not surprising, since now that it is not only ethanol but also water that evaporates the (total) evaporation flux is substantially increased. In particular, this suggests that the quasi-stationary assumption, the condition for which is $P e_{l, \text { diff }} \ll 1$, is less justified when water volatility is taken into consideration. It can even break down for moderate values of $H$, as witnessed by Table 7, although it is still valid for larger $H$. Yet note that the concentration drop across the liquid layer $\left(c_{i, r e f, l}-c_{b}\right)$ does not change any drastically, whereas given the established predominance of the solutal Marangoni mechanism of instability it is $\left(c_{i, r e f, l}-c_{b}\right)$ that is important from the viewpoint of the instability threshold (see also the next subsection). Examining (64) and (104) at $c_{t 1}=c_{t 2}=0$, one can see that the difference in $\left(c_{i, r e f, l}-c_{b}\right)$ between Tables 3 and 7 comes from $\delta_{D} p_{\text {sat } 1}$ effectively moderating the value of $K_{e}$ (cf. the expressions for $\widetilde{K}$ ).

Table 8. Characteristics of the reference state (approximate analysis with water evaporation) for different values of $H$ with $c_{t 1}=c_{t 1 *}$ and $c_{t 2}=0$ (10 wt $\%$ ethanol in water)

\begin{tabular}{lcccccc}
\hline \hline$H$ & $P e_{l, \text { diff }}$ & $P e_{g, \text { diff }}$ & $\begin{array}{c}\left(T_{i, \text { ref }}^{d}-T_{b}^{d}\right) \\
{[K]}\end{array}$ & $\left(c_{i, \text { ref }, l}-c_{b}\right)$ & $\left(c_{i, \text { ref }, g 1}-c_{t 1}\right)$ & $\left(c_{i, r e f, g 2}-c_{t 2}\right)$ \\
& & & & \\
\hline 1.5 & $1.35^{*} 10^{-1}$ & $4.67 * 10^{-3}$ & $-3.62 * 10^{-1}$ & $-8.52 * 10^{-2}$ & $7.83 * 10^{-4}$ & $3.02 * 10^{-3}$ \\
2 & $9.77 * 10^{-2}$ & $6.75^{*} 10^{-3}$ & $-2.43 * 10^{-1}$ & $-7.42 * 10^{-2}$ & $6.86 * 10^{-4}$ & $5.31 * 10^{-3}$ \\
4 & $5.63 * 10^{-2}$ & $1.17 * 10^{-2}$ & $-1.27 * 10^{-1}$ & $-4.87 * 10^{-2}$ & $4.58 * 10^{-4}$ & $1.07 * 10^{-2}$ \\
11 & $2.46 * 10^{-2}$ & $1.70 * 10^{-2}$ & $-5.27 * 10^{-2}$ & $-2.20 * 10^{-2}$ & $2.11 * 10^{-4}$ & $1.66^{*} 10^{-2}$ \\
101 & $3.03 * 10^{-3}$ & $2.10^{-2} 10^{-2}$ & $-6.35^{*} 10^{-3}$ & $-2.73 * 10^{-3}$ & $2.64 * 10^{-5}$ & $2.09 * 10^{-2}$ \\
\hline \hline
\end{tabular}

Adding Table 8 into this discussion shows that choosing the ambient humidity at $c_{t 1}=c_{t 1 *}$ can reduce the effective role of water volatility to a minimum indeed. The values of $\left(c_{i, r e f, l}-c_{b}\right)$ represented by Tables 3 and 8 prove to be nearly identical for all values of $H$ considered. As for the Péclet numbers and the temperature difference, they are close for larger values of $H$, but for moderate $H$ values a certain divergence still persists (but to a much lesser extent than between Tables 3 and 7, without putting the quasi-stationary assumption into question). The reason is that for moderate $H$ values the value of $c_{i, r e f, l}$ becomes appreciably smaller than $c_{b}$, whereas the choice $c_{t 1}=c_{t 1 *}$ performs the best towards the goal of reducing 
water evaporation when $c_{i, r e f, l}$ is not too different from $c_{b}$. Thus, even with $c_{t 1}=c_{t 1 *}$, it is not possible to completely get rid of the effects of water evaporation, although it is possible within certain aspects essential for the present paper.

Note that in a practically important particular case $H \gg 1$, we have $\left|c_{i, r e f, l}-c_{b}\right| \ll c_{b}$, and the results (104)-(108) take a particularly simple form:

$c_{i, r e f, l}-c_{b}=-\frac{\rho D}{H-1} \frac{c_{b}\left(1-c_{b}\right)}{1+c_{b}\left(\delta_{M}-1\right)} \frac{K_{e}-\delta_{D} p_{s a t 1}}{\delta_{M}^{\prime} p_{t}^{d}}+\frac{\rho D}{H-1}\left(1-c_{b}\right) c_{t 2}-\frac{\rho D \delta_{D}}{H-1} c_{b} c_{t 1}$,

$J_{1, \text { ref }}=\frac{\rho D \delta_{D} L e_{l}}{H-1}\left(\frac{1-c_{b}}{1+c_{b}\left(\delta_{M}-1\right)} \frac{p_{s a t 1}}{\delta_{M}^{\prime} p_{t}^{d}}-c_{t 1}\right)$,

$J_{2, \text { ref }}=\frac{\rho D L e_{l}}{H-1}\left(\frac{c_{b}}{1+c_{b}\left(\delta_{M}-1\right)} \frac{K_{e}}{\delta_{M}^{\prime} p_{t}^{d}}-c_{t 2}\right)$,

$T_{i, r e f}-T_{b}=-\left(J_{2, \text { ref }}+\delta_{L} J_{1, \text { ref }}\right)$,

$c_{i, r e f, g 2}=\frac{c_{b}}{1+c_{b}\left(\delta_{M}-1\right)} \frac{K_{e}}{\delta_{M}^{\prime} p_{t}^{d}}, \quad c_{i, r e f, g 1}=\frac{1-c_{b}}{1+c_{b}\left(\delta_{M}-1\right)} \frac{p_{s a t 1}}{\delta_{M}^{\prime} p_{t}^{d}}$,

which is the counterpart of (68)-(71).

\subsection{Stability analysis: Pearson-like model}

The stability analysis is carried out here under the premise that the solutal Marangoni effect remains by far the most important instability mechanism. It is clear however that if water is allowed to evaporate, the thermal effects get stronger (e.g. compare Table 3 and Table 7). Yet the margin between the solutal Marangoni and the other instability mechanisms was so huge here (section 5) that this new circumstance can hardly reverse the overall tendency.

The analysis remains within the approximate approach of sections 6 and 7, when we assume that the convective effects in the gas phase are negligible. As throughout the whole paper, the quasistationary assumption is assumed to hold and besides we neglect the temperature dependence of the Henry coefficient (and now also of the saturation pressure of the solvent). If now the Soret effect is also neglected, the problem for the concentration field decouples from that for the temperature field, and it is in this framework that we keep considering the solutal Marangoni instability.

The counterpart of (72) is now

$C_{g 1}=C_{i, g 1} \frac{\sinh (k(z-H))}{\sinh (k(1-H))}$,

$C_{g 2}=C_{i, g 2} \frac{\sinh (k(z-H))}{\sinh (k(1-H))}$.

The conditions (95) and (97), when rewritten in terms of perturbations (linearization around the reference state) and with the Soret effect neglected in (97), become

$C_{g 1}=-\frac{\delta_{M} C_{l}}{\left[1+c_{l, r e f}\left(\delta_{M}-1\right)\right]^{2}} \frac{p_{s a t 1}}{\delta_{M}^{\prime} p_{t}^{d}}, \quad C_{g 2}=\frac{C_{l}}{\left[1+c_{l, r e f}\left(\delta_{M}-1\right)\right]^{2}} \frac{K_{e}}{\delta_{M}^{\prime} p_{t}^{d}}$,

$\frac{\partial C_{l}}{\partial z}=\rho D\left(1-c_{l, r e f}\right) \frac{\partial C_{g 2}}{\partial z}-\rho D \delta_{D} C_{l, r e f} \frac{\partial C_{g 1}}{\partial z}+\frac{J_{r e f}}{L e_{l}} C_{l}$

at $z=1$, where the second equality (93) for $J$ as applied to the reference state has also been taken into account. With (109)-(112) and (100)-(102), one can derive a single boundary 
condition in terms of $C_{l}$ only. It has the form (73), but the expression for the solutal Biot number is now different from (74):

$B i_{S}(k, H)=\rho D \frac{\left(1-c_{i, r e f, l}\right) K_{e}+c_{i, r e f, l} \delta_{D} \delta_{M} p_{s a t 1}}{\left[1+c_{i, r e f, l}\left(\delta_{M}-1\right)\right]^{2} \delta_{M}^{\prime} p_{t}^{d}} k \operatorname{coth}(k(H-1))-\frac{\rho D}{H-1}\left(\frac{c_{i, r e f, l}}{1+c_{i, r e f, l}\left(\delta_{M}-1\right)} \frac{K_{e}}{\delta_{M}^{\prime} p_{t}^{d}}-c_{t 2}\right)-$

$\frac{\rho D}{H-1} \delta_{D}\left(\frac{1-c_{i, r e f, l}}{1+c_{i, r e f, l}\left(\delta_{M}-1\right)} \frac{p_{\text {sat } 1}}{\delta_{M}^{\prime} p_{t}^{d}}-c_{t 1}\right)$,

where (104) should be used for $c_{i, r e f, l}$. Note that for $H \gg 1$ the solutal Biot number attains the form

$B i_{s}(k)=\rho D \frac{\left(1-c_{b}\right) K_{e}+c_{b} \delta_{D} \delta_{M} p_{s a t 1}}{\left[1+c_{b}\left(\delta_{M}-1\right)\right]^{2} \delta_{M}^{\prime} p_{t}^{d}} k$,

which is the counterpart of (76). Now, it just remains to use the Pearson result (75) for the description of the solutal Marangoni instability in our system.

For the marginal curve in terms of $M s^{*}$, the difference between the considerations without and with water evaporation is thus entirely due to the difference in the expressions for the Biot number, namely between (74) and (113), or between (76) and (114) for $H \gg 1$. The critical Marangoni numbers (as corresponding to the minimum of the marginal curve $M s^{*}=M s^{*}(k)$ ) are provided in Table 9 for a number of values of $H$ and water evaporation regimes with $c_{t 2}=$ 0 . Note that $p_{\text {sat } 1} \equiv 0$ with $c_{t 1} \equiv 0$ formally stands for the limiting case of a non-volatile solvent, and the results of section 6 are recovered from those of the present section.

Table 9. The critical solutal Marangoni number for various $H$ values and various regimes of water evaporation (10 wt $\%$ ethanol in water, $\left.c_{t 2}=0\right)$

\begin{tabular}{cccc}
\hline \hline$H$ & $\begin{array}{c}M s_{c}^{*} \\
\left(p_{\text {sat1 } 1} \equiv 0\right)\end{array}$ & $\begin{array}{c}M s_{c}^{*} \\
\left(c_{t 1}=c_{t 1 *}\right)\end{array}$ & $\begin{array}{c}M s_{c}^{*} \\
\left(c_{t 1}=0\right)\end{array}$ \\
\hline 1.5 & 364.9 & 363.6 & 317.8 \\
2 & 310.8 & 310.5 & 285.2 \\
4 & 303.3 & 304.2 & 295.6 \\
11 & 305.6 & 307.3 & 304.9 \\
101 & 307.3 & 309.5 & 309.3 \\
$\infty$ & 307.5 & 309.8 & 309.8 \\
\hline \hline
\end{tabular}

For each given $H$, there appears to be no big difference in terms of $M s_{c}^{*}$ between the three water evaporation regimes. It is curious however that for smaller $H, c_{t 1}=c_{t 1 *}$ is capable of bringing $M s_{c}^{*}$ appreciably closer (than for larger $\mathrm{H}$ ) to the value obtained while neglecting solvent volatility. On the other hand, in the limit of large $H, M s_{c}^{*}$ ceases to be controlled by the value of $c_{t 1}$, cf. (114). But in this limit, $M s_{c}^{*}$ does not practically depend on the water evaporation regime anyway. A small difference of about $1 \%$ is represented by $c_{b} \delta_{D} \delta_{M} p_{\text {sat } 1}$ versus $\left(1-c_{b}\right) K_{e}$, as one can observe by comparing equations (76) and (114). In view of $c_{b}=0.1$, this is much smaller than the difference between the corresponding reference solutions for $\left(c_{i, r e f, l}-c_{b}\right)$, 
which is represented (for $c_{t 1}=0$ and $c_{t 2}=0$ ) by $\delta_{D} p_{s a t 1}$ versus $K_{e}$ (cf. $\widetilde{K}$ below (64) and (104)). This is why the result for the asymptotic behavior of the critical liquid layer thickness

$\frac{d_{l, c}^{2}}{d_{g}} \approx 1.33 \times 10^{-8} \mathrm{~m} \quad$ as $H \rightarrow \infty \quad\left(c_{t 1}=0, c_{t 2}=0\right)$

differs more from (77) than the corresponding $M s_{c}^{*}$ values in Table 9. On the other hand, the result

$\frac{d_{l, c}^{2}}{d_{g}} \approx 1.03 \times 10^{-8} \mathrm{~m} \quad$ as $\quad H \rightarrow \infty \quad\left(c_{t 1}=c_{t 1 *}, c_{t 2}=0\right)$

is closer to (77). By comparing (115) with (77) or (116), one can observe that water evaporation plays a stabilizing role by increasing the critical liquid thickness.

All in all, we conclude that, given that the solutal Marangoni effect is by far the predominant mechanism of instability here, taking water volatility into account does not result in noteworthy changes in what the instability threshold is concerned. This would of course not be the case in general, should the thermal mechanisms of instability be more important, since the temperature gradient in the reference state is much more sensitive to the regime of water evaporation (likely due to the large latent heat of water). However, for the ambient humidity $c_{t 1}=c_{t 1 *}$, the changes generally turn out to be even less important, and even reduced to a minimum in what the values of the temperature gradients and the evaporation fluxes are concerned (cf. subsection 7.4). This justifies the non-volatile solvent approach used in the previous sections of the present paper in application to a water-ethanol system: it works relatively well in its entirety for $c_{t 1}=c_{t 1 *}$.

\section{Conclusions}

Rayleigh-Marangoni instability in a horizontal layer of a dilute binary liquid mixture evaporating into air from its flat free surface has been studied. An aqueous solution of ethanol $(10 \mathrm{wt} \%$ at $300 \mathrm{~K}$ ) has been used as the principal example treated throughout the paper, even though the case of $0.1 \% \mathrm{wt}$ has also been tested. The primary goal was to analyze the relative importance of various instability mechanisms. For this reason, a particularly simple configuration, with the concentration fixed at the bottom, yet admitting a (quasi-)stationary reference solution has been considered (the "quasi-stationarity" here referring to sufficiently slow evolution of the liquid layer thickness as compared to other time scales of the problem). The transfer in the gas has been modeled by specifying a transfer distance entering into the model as the gas layer thickness. Such an approach is more general than the typically used one based upon a one-layer model with a constant transfer coefficient (Biot number) at the free surface, for it potentially permits to account for a variety of possible convective effects in the gas phase as well as for the dependence of the effective transfer coefficient on the wavenumber of the perturbation. It is natural, identifying this transfer distance with an effective boundary-layer thickness, to impose given ("ambient") temperature and composition at the artificial top boundary of the gas layer (here we assumed that the temperature was equal to that of the bottom of the liquid layer and that no ethanol vapor was present in the external air). The choice of the hydrodynamic conditions, however, is not so obvious, and this question has also been explored in this paper. By default, we 
imposed a fixed normal stress and a zero tangential stress, which anyway has turned out to have only little influence on the results.

At first, the analysis has been developed based on the premise that it is only the solute (ethanol) that evaporates whereas the solvent (water) does not (as detailed below, this situation can be approached by imposing the right ambient humidity of the air). At a later stage, the solvent (water) evaporation has also been incorporated into the model, permitting an instructive comparison with the former case. As for air absorption in the liquid, it has been neglected throughout the paper. After selecting the liquid (viz. $10 \mathrm{wt} \%$ ethanol in water), the main control parameters we are left with are the thicknesses of the liquid and gas layers. Thus, the critical condition for the onset of instability (which proves to be monotonic in this case) is expressed in terms of the critical liquid thickness as a function of the gas layer thickness. The former increases with the latter (asymptotically more or less as a square root). The system proves to be extremely unstable, and the resulting critical liquid thicknesses are very small (e.g. about $1 \mu \mathrm{m}$ for a gas layer thickness of $0.1 \mathrm{~mm}$ ). The dimensionless critical wavenumber always remains more or less the same (about 2 in the units of the inverse liquid layer thickness), as in the classical Bénard problem (cellular convection).

An inquiry into the physical nature of the computed results reveals that it is the solutal Marangoni effect that is primarily responsible for the instability, and is by far the strongest. The solutal Rayleigh and the thermal Marangoni and Rayleigh effects all prove to be small at the instability threshold. The overall influence of the Soret effect in the liquid also remains minimal: for situations considered here, it is of the same order of magnitude as the thermal Marangoni and Rayleigh effects.

After having established that the solutal Marangoni effect is the principal instability mechanism, further physical details of the phenomenon have been studied. In particular, the smallness of Péclet numbers indicates a small evaporation flux, which shows that the latter plays only a minor convective role, even though creating the basic concentration (and temperature) gradients responsible for the instability. On the basis of this observation, and neglecting further the dynamic viscosity of the gas, a one-layer model has been derived, with the mass transport in the gas phase being accounted for by means of an effective solutal Biot number depending on the wavenumber of the perturbation and on the ratio of the gas and liquid layer thicknesses. With this expression of the Biot number, one can use the Pearson result for the neutral curve in terms of the Marangoni number (solutal in our case) versus the wavenumber. An excellent agreement of this Pearson-like model with the results of a "full analysis" has been demonstrated in the paper, which confirms the validity of the above mentioned physical conjectures. We note that under one of the conditions listed above (viz. small Peclet numbers), the problem for the basic (reference) profile can readily be resolved analytically (with or without water evaporation), which together with the Pearson-like model yields a fully analytical approximate result for the instability threshold in a wide range of parameter values. 
The success of the Pearson-like model partly explains why only a slight dependence of the results of the full analysis on the hydrodynamic boundary conditions posed at the top boundary of the gas layer has been observed: the hydrodynamics in the gas phase plays just a minor role. Nonetheless, for very small gas layer thicknesses, of the order of one half of the corresponding critical liquid layer thickness, it has been numerically observed that the hydrodynamic conditions start to make a difference (e.g. replacing a zero normal stress condition by a zero normal velocity one within the problem for perturbations produces a noticeable deviation in the results and the Pearson-like model ceases to work).

Additionally incorporating evaporation of the solvent (water) into the model (which here has been done just in the framework of the approximate solution for the reference profile, as described above, and the Pearson-like approach relying on the supremacy of the solutal Marangoni mechanism of instability) does not produce much of a change in what the concentration difference across the liquid layer and the instability threshold are concerned. This of course increases the corresponding temperature difference and the total evaporation mass flux (about five times for the former and three times for the latter in the studied example if the ambient air is completely dry and for $d_{g} / d_{l}>>1$, where $d_{g}$ and $d_{l}$ are the gas and liquid layer thicknesses). Yet this is insufficient to inverse the tendency for the thermal Rayleigh and Marangoni effects to be much weaker than the solutal Marangoni effect, for the Soret effect to be insignificant and for the evaporation Péclet numbers to remain small, even though a three-fold increase in the evaporation flux (and thus the Péclet numbers) should somewhat deteriorate the quality of the Pearson-like model. For a practically less important (in view of very small critical values of $d_{l}$ ) case of moderate values of $d_{g} / d_{l}$, however, the total evaporation flux and the temperature difference increase more drastically than mentioned above. While still far from challenging the supremacy of the solutal Marangoni effect, these alterations can put into question some assumptions used in the model here, such as e.g. the smallness of the Péclet number in the liquid, which ensures the validity of the quasi-stationary assumption used throughout the paper. Interestingly enough, for large values of $d_{g} / d_{l}$, the result for the neutral curve in terms of the solutal Marangoni number versus the wavenumber (and the Biot number), as established here within the Pearson-like model, is not practically modified should the water be allowed to evaporate. Yet the critical liquid layer thickness undergoes a stronger change (about $15 \%$ for dry ambient air), and this is primarily due to the corresponding change in the concentration gradient across the liquid layer in the reference state. It has also been shown that for a certain "right" ambient humidity of the air (i.e. close to that corresponding to thermodynamic equilibrium at the given liquid composition and imposed total pressure and temperature), the considerations with and without water evaporation produce rather close results even in what concerns the temperature difference and the evaporation flux (even though, once again, we obtain a bigger discrepancy for moderate values of $d_{g} / d_{l}$ ). In principle, this justifies the application of a nonvolatile solvent approach (upon which the main body of the present paper is based) to a waterethanol system, provided that the latter is subjected to this right ambient humidity. 
Given that the system has proved to be highly unstable by means of the solutal Marangoni mechanism, resulting in extremely small critical liquid layer thicknesses, for a realistically thick (say, $1 \mathrm{~mm}$ ) layer of an evaporating binary liquid the instability should occur shortly after its exposure to air, when the transient diffusional layer developing from the free surface due to evaporation has clearly not yet reached the bottom of the liquid. Thus, it is of high interest to study the instability for such a transient reference profile, and this is where future work should be concentrated. On the other hand, this transient character implies a separation of spatial scales which is not accounted for in the framework of the configuration considered in the present paper, and thus further motivates such a prospective study: first of all this concerns the thickness of the developing diffusional layer versus the thickness of the entire liquid layer. Furthermore, due to a small Lewis number, the transient thermal layer will penetrate much deeper than the diffusional one, thus increasing the chances for the thermal Marangoni and Rayleigh effects to get more noticeable versus the solutal ones as the instability mechanisms. The Soret effect could get more noticeable too. The Rayleigh effects may also perform differently given such a separation of scales.

\section{Acknowledgements}

The authors gratefully acknowledge the financial support of the European Space Agency and of the Belgian Science Policy through the PRODEX-CIMEX and PRODEX-BOILING projects. This research was also partially supported by the ARCHIMEDES project funded by the "Communauté Française de Belgique". AR and PC have also beneficiated from discussions with Frédéric Doumenc and Béatrice Guerrier during visits at the FAST laboratory in Orsay, made possible thanks to the MULTIFLOW Marie Curie FP7-ITN Network of the European Union. PC gratefully acknowledges financial support of the Fonds de la Recherche Scientifique - FNRS.

\section{Appendix A: Physical quantities}

The developed model is applied to a binary mixture of ethanol and water, where ethanol is the evaporating solute with a bottom mass fraction of 0.1 or 0.001 , whilst the mass fraction of ethanol is equal to 0 at the top of the gas layer. In order to analyze the stability of this system it is necessary to know the values of the material properties.

\section{A.l Gas layer}

Given the small concentrations of solvent and of solute, the gas properties are taken as those of pure air at $300 \mathrm{~K}$ [30]. Besides, the diffusion coefficient for the ethanol vapor is taken to be that of a "near-zero" mass fraction of ethanol vapor in air $\left(1.20 * 10^{-5} \mathrm{~m}^{2} / \mathrm{s}[31]\right)$, whereas the value for the diffusion coefficient for water vapor is taken from [32] p. 6-213. Furthermore, the values for the heat of evaporation of water $\left(L_{1}\right)$ and the saturation pressure of water vapor are taken from [32] p. 6-4 and p. 6-11 respectively. The solutal expansion coefficient is calculated from the following considerations.

Assuming an ideal gas, we have 
$\frac{p_{g}^{d}}{R T^{d}}=n_{g, a}+n_{g 1}+n_{g 2}, \quad n_{g, a}=\rho_{g}\left(1-c_{g 1}-c_{g 2}\right) \frac{1}{M_{a}}, \quad n_{g 1}=\rho_{g} c_{g 1} \frac{1}{M_{1}}, \quad n_{g 2}=\rho_{g} c_{g 2} \frac{1}{M_{2}}$,

where $n_{g, a}, n_{g 1}$ and $n_{g 2}$ are the air, water and ethanol molar concentrations (moles per unit volume) in the gas phase, while $c_{g 1}$ and $c_{g 2}$ are the mass fractions of the water and ethanol vapors. Substituting the last three expressions into the first one, one obtains

$\rho_{g}=M_{a} \frac{p_{g}^{d} / R T^{d}}{1+\left(\delta_{M}^{\prime}-1\right) c_{g 1}+\left(\delta_{M}^{\prime \prime}-1\right) c_{g 2}}$,

and thus

$\frac{1}{\rho_{g}} \frac{\partial \rho_{g}}{\partial c_{g 1}}=-\frac{\delta_{M}^{\prime}-1}{1+\left(\delta_{M}^{\prime}-1\right) c_{g 1}+\left(\delta_{M}^{\prime \prime}-1\right) c_{g 2}}$

$\frac{1}{\rho_{g}} \frac{\partial \rho_{g}}{\partial c_{g 2}}=-\frac{\delta_{M}^{\prime \prime}-1}{1+\left(\delta_{M}^{\prime}-1\right) c_{g 1}+\left(\delta_{M}^{\prime \prime}-1\right) c_{g 2}}$.

Thus, in view of equation (78), with $c_{g 1,0}=c_{g 2,0}=0$, one can deduce

$\varepsilon_{g 1} \approx \delta_{M}^{\prime}-1$,

$\varepsilon_{g 2} \approx \delta_{M}^{\prime \prime}-1$,

which yields the values shown in Tables 1 and 6 . Here note that in the first part of the paper, where the solvent volatility is neglected, the quantities without subscripts 1 and 2 are associated with the solute, e.g. $\varepsilon_{g} \equiv \varepsilon_{g 2}$ (cf. also equations (2) and (78)).

\section{A.2 Liquid layer}

The physical properties in the liquid are taken at the concentration and temperature of the bottom of the liquid layer, here chosen to be either 0.1 or 0.001 for the mass fraction of ethanol in water and $300 \mathrm{~K}$ for the temperature. In the case of a mass fraction of 0.001 , the physical properties are taken to be those of pure water, with the exception of inherently binary-mixture properties such as $D_{T, l} / D_{l}, D_{l}, \varepsilon_{l}$ and $\gamma_{C}$.

These are taken for a mass fraction of 0.001 of ethanol in water, found in respectively [33], [24] and [34] (p. 2-112). So these values need no further explanation. The values corresponding to a mass fraction of 0.1 are explained in this section. The values are presented in Table 2.

The density $\rho_{l}$, the thermal expansion coefficient $\alpha_{l}$ and the solutal expansion coefficient $\varepsilon_{l}$ are derived from the density tables found in the literature [34] (p. 2-112), where an elementary difference scheme is used to calculate the expansion coefficients. Interpolation is used wherever appropriate. The dynamic viscosity $\mu_{l}$ is derived from [35]. Using Fig. 5 of [35] for an ethanol mass fraction of 0.1 (corresponding to a ratio of about 23 in the number of water to ethanol molecules), one obtains $1.5^{*} 10^{-3} \mathrm{~Pa}^{*} \mathrm{~s}$ for the dynamic viscosity. However, the figure corresponds to $293 \mathrm{~K}$, whereas the value we need here should be for $300 \mathrm{~K}$. Following the work of McMoughlin et al. [36], it is roughly estimated that the dynamic viscosity of a mixture of ethanol and water at $300 \mathrm{~K}$ is about $15 \%$ lower than at $293 \mathrm{~K}$. The value is changed accordingly, as presented in Table 2 . The kinematic viscosity $v_{l}$ is then calculated by dividing the dynamic viscosity by the liquid density. 
The specific heat capacity $c_{p, l}$ of the liquid can be found in [34] (p. 2-184). The thermal conductivity $\lambda_{l}$ of the liquid is extracted from [37] using interpolation/extrapolation, whereas the thermal diffusivity $\kappa_{l}$ is calculated by dividing the thermal conductivity by the liquid density and specific heat. The mutual diffusion coefficient $D_{l}$ is taken from [24]. The properties $\gamma_{T}$ and $\gamma_{C}$ are derived from [38] using interpolation/extrapolation. Henry's coefficient is taken according to Warneck [39] as

$K_{e}\left(T^{d}\right)=K_{e}(298.15) e^{\left[-\frac{L^{(m o l e)}}{R}\left(\frac{1}{T^{d}(K)}-\frac{1}{298.15}\right)\right]}$,

where $L^{(m o l e)}$ is the molar heat of solution of ethanol vapor in water, $R$ the universal gas constant and $K_{e}(298.15)=0.286 \mathrm{~atm}$. The value of the heat of solution is shown in Table 2 as given by $L=L^{(\text {mole })} / M_{2}$, where $M_{2}$ is the molar mass of ethanol.

The Soret effect is neglected in the gas layer, but not in the liquid layer. The Soret coefficient is approximated by the following expression [33]:

$\frac{D_{T, l}}{D_{l}}=10^{-3}\left(2.493-\frac{T^{d}\left({ }^{\circ} \mathrm{C}\right)}{39.661}\right)\left(1-3.190\left[\tanh \left(\frac{c_{l}-0.2387}{0.1668}\right)^{3}\right]^{1 / 3}\right)$.

Taking $c_{l}=0.1$ or 0.001 and $T_{d}=27^{\circ} \mathrm{C}$ here yields the values shown in Table 2 .

\section{Appendix B: Nomenclature}

Note that for some symbols, the units indicated pertain to the dimensional form of the corresponding quantity (distinguished by the superscript "d"), while the corresponding quantity without subscript " $\mathrm{d}$ " is dimensionless.

$\begin{array}{ll}\text { Symbols } & \text { Unit vector in the z-direction } \\ 1_{z} & \text { Mass fraction [-] } \\ c & \text { Heat capacity at constant pressure }[\mathrm{J} / \mathrm{kg} \mathrm{K}] \\ c_{p} & \text { Layer thickness }[\mathrm{m}] \\ d & \text { Diffusion coefficient }\left[\mathrm{m}^{2} / \mathrm{s}\right] \\ D & \text { Thermal diffusion coefficient }\left[\mathrm{m}^{2} / \mathrm{s} \mathrm{K}\right] \\ D_{T} & \text { Gravitational acceleration }\left[\mathrm{m} / \mathrm{s}^{2}\right] \\ g & \text { Galileo number } \\ G a & \text { Mass flux }\left[\mathrm{kg} / \mathrm{m}^{2} \mathrm{~s}\right] \text { (or dimensionless) } \\ J & \text { Wavenumber }\left[\mathrm{m}^{-1}\right](\text { or dimensionless) } \\ k & \text { Henry's coefficient of the solute }[\mathrm{atm}] \\ K_{e} & \text { Latent heat of phase change (heat of solution for the solute) } \\ L & {[\mathrm{~J} / \mathrm{kg}]} \\ & \text { Lewis number } \\ L e & \text { Molecular weight }[\mathrm{kg} / \text { mole }] \\ M & \text { Thermal Marangoni number } \\ M a & \text { Solutal Marangoni number } \\ M S & \text { Pressure [Pa] (or dimensionless) } \\ p & \end{array}$




\begin{tabular}{|c|c|}
\hline $\operatorname{Pr}$ & Prandtl number \\
\hline$q$ & Heat flux $\left[\mathrm{W} / \mathrm{m}^{2}\right]$ (or dimensionless) \\
\hline$R$ & Universal gas constant $[\mathrm{J} /$ mole $\mathrm{K}]$ \\
\hline$R a$ & Thermal Rayleigh number \\
\hline Rs & Solutal Rayleigh number \\
\hline$S_{l, 0}$ & Soret coefficient $\left[\mathrm{K}^{-1}\right]$ \\
\hline$T$ & Temperature $[\mathrm{K}]$ (or dimensionless) \\
\hline$u, v, w$ & Velocity in $\mathrm{x}, \mathrm{y}$ and $\mathrm{z}$ directions $[\mathrm{m} / \mathrm{s}]$ (or dimensionless) \\
\hline$x, y, z$ & Cartesian coordinates [m] (or dimensionless) \\
\hline \multicolumn{2}{|c|}{ Greek letters } \\
\hline$\alpha$ & Thermal expansion coefficient $\left[\mathrm{K}^{-1}\right]$ \\
\hline$\gamma$ & Surface tension $[\mathrm{N} / \mathrm{m}]$ \\
\hline$\gamma_{C}$ & $\begin{array}{l}\text { Sign-changed derivative of surface tension with respect to } \\
\text { mass fraction }[\mathrm{N} / \mathrm{m}]\end{array}$ \\
\hline$\gamma_{T}$ & $\begin{array}{l}\text { Sign-changed derivative of surface tension with respect to } \\
\text { temperature }[\mathrm{N} / \mathrm{m} \mathrm{K}]\end{array}$ \\
\hline$\delta$ & $\begin{array}{l}\text { Ratio of solvent and solute properties, the subscript of } \delta \\
\text { indicating the property in question }\end{array}$ \\
\hline$\delta^{\prime}$ & $\begin{array}{l}\text { Ratio of air and solvent properties, the subscript of } \delta^{\prime} \\
\text { indicating the property in question }\end{array}$ \\
\hline$\delta^{\prime \prime}$ & $\begin{array}{l}\text { Ratio of air and solute properties, the subscript of } \delta^{\prime \prime} \\
\text { indicating the property in question }\end{array}$ \\
\hline$\varepsilon$ & Solutal expansion coefficient [-] \\
\hline$\theta$ & Temperature scaling factor $[\mathrm{K}]$ \\
\hline$\kappa$ & Thermal diffusivity $\left[\mathrm{m}^{2} / \mathrm{s}\right]$ \\
\hline$\lambda$ & Thermal conductivity $[\mathrm{W} / \mathrm{m} \mathrm{K}]$ \\
\hline$\mu$ & Dynamic viscosity $[\mathrm{Pa} \mathrm{s}]$ \\
\hline$v$ & Kinematic viscosity $\left[\mathrm{m}^{2} / \mathrm{s}\right]$ \\
\hline$\rho$ & Density $\left[\mathrm{kg} / \mathrm{m}^{3}\right]$ \\
\hline$\sigma$ & Complex growth rate $\left[\mathrm{s}^{-1}\right]$ (or dimensionless) \\
\hline$\psi_{S}$ & Soret number \\
\hline \multicolumn{2}{|c|}{ Subscripts } \\
\hline 0 & Reference point \\
\hline 1 & Solvent \\
\hline 2 & Solute \\
\hline$a$ & Air \\
\hline$b$ & Bottom plate \\
\hline
\end{tabular}


No subscript

Superscript

$d$

$n$

$*$
Ethanol

Gas phase (air + vapor)

Interface

Liquid phase (water + ethanol)

Reference state

Saturation

Top boundary

With the material property symbols, it designates the gasto-liquid ratio of the corresponding property, e.g.:

$\rho=\rho_{g} / \rho_{l}$.

Superscript " $d$ " stresses that a quantity is dimensional (for instance, $T^{d}$ is the dimensional temperature, while $T$ is the corresponding dimensionless quantity). This superscript is not applied to dimensional quantities that are never used in dimensionless form anyway.

It designates the non-dimensional form of certain dimensional quantities.

Designating the "true" Marangoni and Rayleigh numbers (classical definitions based upon the temperature or concentration differences across the liquid layer)

\section{References}

[1] H. Bénard, Ann. Chem. Phys. 23 (1901) 62.

[2] D.A. Nield, J. Fluid Mech. 19 (1964) 341.

[3] J.R.A. Pearson, J. Fluid Mech. 4 (1958) 489.

[4] E.L. Koschmieder, Bénard Cells and Taylor Vortices, Cambridge Univ. Press, Cambridge, 1993.

[5] C. Normand, Y. Pomeau, M. Velarde, Rev. Mod. Phys. 49 (1977) 581.

[6] P. Colinet, J.C. Legros, M.G. Velarde, Non Linear Dynamics of Surface Tension Driven Instabilities, Wiley-VCH Verlag, Berlin, 2001.

[7] P. Bergé, M. Dubois, Contemp. Phys. 25 (1984) 535.

[8] P.B. Drazin, W. Reid, Hydrodynamic Stability, Cambridge Univ. Press, Cambridge, 1981.

[9] P. Colinet, L. Joannes, C.S. Iorio, B. Haut, M. Bestehorn, G. Lebon and J.C. Legros, Adv. Space Res. 32 (2003) 119.

[10] B. Haut, P. Colinet, J. Colloid Interface Sc. 285 (2005) 296.

[11] M. Dondlinger, J. Margerit, PC. Dauby, J. Colloid Interface Sc. 283 (2005) 522.

[12] O. Ozen O, R. Narayanan, Ind. Eng. Chem. Res. 44 (2005) 1342. 
[13] J. Margerit, M. Dondlinger, PC. Dauby, J. Colloid Interface Sc. 290 (2005) 220.

[14] J. Margerit, P. Colinet, G. Lebon, CS. Iorio, JC. Legros, Physical Review E. 68 (2003) 041601.

[15] O. Ozen O, R. Narayanan, Physics of Fluids 16 (2004) 4644.

[16] P.G. de Gennes, Eur. Phys. J. E. 6 (2001) 421.

[17] G. Toussaint, H. Bodiguel, F. Doumenc, B. Guerrier, C. Allain, Int. J. Heat Mass Transfer 51 (2008) 4228.

[18] V.-M. Ha, C.-L. Lai, Int. J. Heat Mass Transfer 45 (2002) 5143.

[19] N. Kurenkova, K. Eckert, E. Zienicke, A. Thess, Lect. Notes Phys. 628 (2003) 403.

[20] S. Dehaeck, C. Wylock, P. Colinet, Phys. Fluids 21 (2009) 091108.

[21] R.B. Bird, W.E. Stewart, E.N. Lightfoot, Transport Phenomena, John Wiley \& Sons, New York, 1960.

[22] P. Atkins, Physical Chemistry: Chapter Simple Mixtures, Oxford University Press, Oxford, 2001.

[23] M. Dondlinger, P. Colinet, PC. Dauby, Physical Review E. 68 (2003) 066310.

[24] K.C. Pratt and W.A. Wakeham, Proc. R. Soc. Lond. A 336, No.1606 (1974) 393.

[25] G.J.M. Pieters and C.J. van Duijn, European Journal of Mechanics - B/Fluids 25 (2006) 83.

[26] L. Czechowsk and K. Kossacki, Icarus, doi:10.1016/j.icarus.2009.02.032, 2009.

[27] P.M. Parmentier, V.C. Regnier, G. Lebon, J.C. Legros, Physical Review E. 54 (1996) 411.

[28] J.P. Burelbach, S.G. Bankoff and S.H. Davis, J. Fluid Mech. 195 (1988) 463.

[29] S.J. VanHook, M.F. Schatz, W.D. McCormick, J.B. Swift, H.L. Swinney, Phys. Rev. Letters 75 (1995) 4397.

[30] http://www.engineeringtoolbox.com/air-properties-d_156.html, 2009.

[31] D.G.F.Naterer, Heat transfer in single and multiphase systems p. 593. CRC Press, 2003.

[32] D.R. Lide (Ed.), CRC Handbook of Chemistry and Physics, CRC Press, 2008.

[33] J.K. Platten, J.F. Dutrieux, G. Chavepeyer, in: W. Köhler, S. Wiegand (Eds.), Thermal Nonequilibrium Phenomena in Fluid Mixtures, Springer, 2000, 313.

[34] R.H.Perry, D.W.Green, J.O.Maloney (Eds.), Perry's Chemical Engineers' Handbook, McGraw-Hill, 1997, 2-112.

[35] M.Ageno, C.Frontali, Proceedings of the National Academy of Sciences 57, No.4 (1967) 856.

[36] N.McMoughlin, S.L.Lee and G.Hähner, Appl. Phys. Lett. 89 (2006) 184106(1-3).

[37] M.J.Assael, E.Charitidou, W.A.Wakeham, Int. J. Thermophys. 10, No.4 (1989) 793.

[38] R.C.Weast, S.M.Selby, C.D.Hodgman (Eds.), Handbook of Chemistry and Physics, The Chemical Rubber Co, 1964, F-18.

[39] P.Warneck, Atmospheric Environment 40, N.37 (2006) 7146. 


\section{Figure captions}

Fig. 1. Sketch of the studied system

Fig. 2. Temperature distribution in the reference state (10 wt $\%$ ethanol in water)

Fig. 3. Solute mass distribution in the reference state (10 wt $\%$ ethanol in water)

Fig. 4. Neutral stability curve in terms of the liquid layer thickness for $H=2,11$ and 101 (10 $\mathrm{wt} \%$ ethanol in water)

Fig. 5. The critical liquid thickness as a function of $H(a)$ and $d_{g}$ (b) for an ethanol/water system with $c_{b}=0.1$ (10 wt\% ethanol in water), and the same, albeit in different scales, together with the results for $c_{b}=0.001(0.1 \mathrm{wt} \%$ ethanol in water $)(\mathrm{c}, \mathrm{d})$

Fig. 6. The ratios of the solutal and the Soret contributions as a function of $d_{g}(10 \mathrm{wt} \%$ ethanol in water)

Fig. 7. The marginal stability curve in terms of the "true" solutal Marangoni number comparing the full analysis with the consideration of the solutal Marangoni effect only for $H=2$ (a) and $H=$ 101 (b) in the case of an ethanol/water mixture of 10/90 wt $\%$

Fig. 8. The "true" solutal Marangoni number as a function of the wavenumber at the marginal condition for different values of $H$ in the case of an ethanol/water mixture of 10/90 wt \%

Fig. 9. The critical solutal Marangoni number $M s^{*}$ as a function of the gas layer thickness for an ethanol/water mixture of $10 / 90 \mathrm{wt} \%$

Fig. 10. The critical solutal Marangoni number as a function of the gas layer thickness for different kinds of hydrodynamic boundary conditions at the top boundary (10 wt $\%$ ethanol in water)

Fig. 11. The marginal curves in terms of the "true" solutal Marangoni number versus the wave number: comparison between the complete model of the present paper and the Pearson-like simplified model for $\mathrm{H}=1.5$ (a), $\mathrm{H}=2$ (b), $\mathrm{H}=11$ (c) $\mathrm{H}=101$ (d) (10 wt\% ethanol in water)

Fig. 12. The critical liquid thickness (a) and the critical "true" solutal Marangoni number (b) versus the gas layer thickness: comparison with the Pearson-like model $(10 \mathrm{wt} \%$ ethanol in water) 